\title{
Computation of the Large Scale Vertical Velocity ${ }^{1}$
}

\author{
G. J. Haltiner, \\ U. S. Naval Postgraduate School, Monterey, Calif. \\ I. C. Claarke and G. E. Lawniczak, JR., LT., USN \\ Fleet Numerical Weather Facility, Monterey, Calif.
}

(Manuscript received 23 August 1962, in revised form 20 November 1962)

\begin{abstract}
Vertical velocities are computed at four levels over a Northern Hemisphere grid in addition to a lower boundary value which is applied at terrain pressure and includes effects of frictionally- and terrain-induced vertical velocities. The latter, as computed here, have somewhat smaller maximum magnitudes and less irregularity than the frictionally-induced vertical velocity.

The calculations show that the terrain and friction effects markedly influence the $\omega$-fields in the lower troposphere but largely disappear by $500 \mathrm{mb}$ for the rather heavily smoothed mountains used in these experiments.

Computations with several static stability parameters, namely a constant value, a value varying with pressure only, and a point-variable value, exhibit the greatest differences in the maximum vertical velocities, as much as 50 per cent, at $300 \mathrm{mb}$. At lower altitudes the differences are only about 10 per cent.

Similarly when computations were made utilizing the term $f \eta$ versus $f_{M^{2}}$ in the coefficient of $\partial^{2} \omega / \partial p^{2}$ in the $\omega$-equation, differences in $\omega$ up to 50 per cent occurred; but mostly the differences were only 10 to 25 per cent.

The computed vertical velocities during a severe west coast storm appear to show good correlation with the weather.
\end{abstract}

\section{Introduction}

The importance of vertical velocity with respect to the development and dissipation of clouds and precipitation is well known. Moreover, synoptic forecasters are becoming increasingly aware of the usefulness of the vertical velocity field with respect to prediction of largescale features of the pressure field (Burnett, 1961).

There have been several more or less important methods for obtaining estimates of the large-scale vertical wind velocity from conventional meteorological observations, for example, the "adiabatic," "kinematic," and "vorticity" methods (see, for example, Haltiner and Martin, 1957). The first of these is based on the first law of thermodynamics and utilizes observed local temperature changes, advective changes and static stability to compute the vertical velocity. The main disadvantage here appears to lie in the reliance on 12-hourly upper air observations to estimate local time derivatives as well as the horizontal advection. In the kinematic method the continuity equation is used and the horizontal wind velocity divergence is integrated vertically to yield the vertical velocity. Unfortunately the large-scale velocity divergence is difficult to obtain directly from routine observations.

1 This research was supported in part by the Office of Naval Research.
In recent years with the application of the electronic computer to numerical forecasting, the vorticity method has gained the ascendency. In its simpler forms, this method consists in eliminating local time derivatives of vorticity and temperature from the vorticity equation and the first law of thermodynamics (thermal equation) ${ }^{2}$ leaving a "diagnostic" second order partial differential equation in the vertical velocity which may be solved from known velocity and temperature fields.

Such investigations of the vertical velocity field previous to this one have generally been more limited in scope with respect to either (a) horizontal area, (b) vertical resolution, or (c) the various approximations utilized in obtaining the solution. In connection with (a) and (b), the selection of levels here was governed primarily by the availability of machine-analyzed hemispheric pressure-height and temperature data. At the time of this investigation the U. S. Navy Fleet Numerical Weather Facility was providing, on an operational basis, twice-daily objective analysis of the $z$ and $T$ fields over the Northern Hemisphere at the 1000-, 850-, 700-, 500and $300-\mathrm{mb}$ levels. It was particularly desired to obtain the vertical velocities at these levels so that they could be directly correlated with other parameters such as

${ }^{2}$ An exact form requires the use of the vorticity, divergence continuity and thermal equations as well as the hydrostatic and
state equations. 
pressure-height, temperature and also static stability. This necessitated the use of unequally spaced pressure intervals in the computation of the vertical derivatives, but gave the model some flexibility if additional levels became available or the levels were changed.

With regard to (c) above, the lower-boundary condition was of special interest in devising the model. In most previous studies, the vertical velocity at the lower boundary was assumed zero or computed from terrainfriction effects and applied at the lowest pressure level in the model, usually $1000 \mathrm{mb}$. Then the omega-equation was integrated with complete disregard of the presence of the terrain features. In this study the vertical velocity for the lower boundary is computed with both mountain and friction effects at the pressure level of the terrain and applied there. Since the pressure at terrain-height varies horizontally, unequally spaced pressure intervals are again necessary in computing vertical derivatives and this spacing varies from point to point. Moreover, in the integration procedure, points within the terrain on any pressure surface constitute an internal boundary and are not relaxed. The procedures used in this connection will be described in detail in later sections.

\section{Theoretical development}

The vorticity and thermal equations for adiabatic frictionless flow may be written, respectively, in the forms

$$
\begin{gathered}
\frac{\partial \zeta}{\partial t}+\mathbf{V} \cdot \nabla(\zeta+f)+\omega-\frac{\partial \zeta}{\partial p}=\underset{\eta}{\partial p}+\frac{\partial \omega}{\partial y} \frac{\partial \omega}{\partial p}-\frac{\partial \omega}{\partial x} \frac{\partial v}{\partial p} \\
\frac{\partial T}{\partial t}+\mathbf{V} \cdot \nabla T-\sigma \omega=0,
\end{gathered}
$$

where

$$
\sigma=\frac{R T}{p c_{P}}-\frac{\partial T}{\partial p} .
$$

The symbols used here are standard. At this point the local change of vorticity in (1) is approximated by its geostrophic counterpart, namely,

$$
\frac{\partial \zeta}{\partial t} \doteq \frac{g}{f} \nabla^{2} \frac{\partial z}{\partial t}
$$

Next, (1) is operated on with $\partial / \partial p, \mathrm{Eq}(2)$ with $\nabla^{2}$. Use of the hydrostatic approximation

$$
\frac{\partial z}{\partial p}=-\frac{R T}{g p}
$$

in the local time derivative of temperature permits the elimination of the time derivatives between (1) and (2), leaving a diagnostic equation in $\omega$.

$$
\begin{aligned}
\nabla^{2}(\sigma \omega)+ & \frac{p f \eta}{R} \frac{\partial^{2} \omega}{\partial p^{2}}+\frac{p f}{R} \frac{\partial}{\partial p}\left(\frac{\partial \omega}{\partial y} \frac{\partial u}{\partial p}-\frac{\partial \omega}{\partial x} \frac{\partial v}{\partial p}\right) \\
& -\frac{\omega p f}{R} \frac{\partial^{2} \zeta}{\partial p^{2}}-\nabla^{2}(\mathbf{V} \cdot \nabla T)-\frac{p f}{R} \frac{\partial}{\partial p} V \cdot \nabla \eta=0
\end{aligned}
$$

The third and fourth terms arise from the vertical advection of vorticity and the twisting term in the vorticity equation. The latter are usually considered to be not only of smaller magnitude than the remaining terms, but also themselves of opposite sign, and thus are frequently neglected in application of the vorticity equation. Hence the third and fourth terms of (6) will be omitted here; however they are to be considered in some subsequent computations.

The first term of (6) may be expanded to give

$$
\nabla^{2}(\sigma \omega)=\sigma \nabla^{2} \omega+\omega \nabla^{2} \sigma+2 \nabla \sigma \cdot \nabla \omega .
$$

Some preliminary theoretical estimates of the three terms comprising (7) indicated that the last two terms are somewhat smaller than the first. Hence only the first term was included in the initial computations, however the other terms were included later.

At this point it should be indicated that the wind and vorticity are to be further approximated at this stage in the project by their geostrophic counterparts. Now for the time-integration of $\mathrm{Eq}(1)$ and (2) in the geostrophic case (vertical advection and twisting terms omitted), certain consistency conditions regarding energy and the mean generation of vorticity (Lorenz, 1960; WiinNielsen, 1959), strictly speaking, require that the absolute vorticity in the divergence term be approximated by mean value of the coriolis parameter, and also that the static stability $\sigma$ be treated as a constant or at most a function of $p$. Only a diagnostic solution is being sought here, without any integration with respect to time. Nevertheless for partially evaluating the effect of a variable $\sigma$, the program has been designed to compute the $\omega$-field with not only a constant mean static stability $\overline{\bar{\sigma}}$, as determined by the observational data, but also a mean value $\bar{\sigma}$ for each pressure level and a point-variable $\sigma$. Similarly the coefficient $f \eta$ in the second term of (6) has been alternated with the mean value $f_{M}{ }^{2}$, again for partially evaluating the effect of the variable coefficient $\eta$ in the divergence term of the vorticity equation. The above variations could be included in the program readily; whereas the inclusion of complete vorticity equation, as well as all three terms of (7), would have taxed the computer system's storage capacity and lengthened the time for solution to the point of rendering the program impractical for operational use. With the various approximations mentioned above, (7) re- 
duces to

$$
\sigma \nabla^{2} \omega+\frac{p f \eta}{R} \frac{\partial^{2} \omega}{\partial p^{2}}=\nabla^{2}(\mathbf{V} \cdot \nabla T)+\frac{p f}{R} \frac{\partial}{\partial p}(\mathbf{V} \cdot \nabla \eta) .
$$

Expanding the derivative of the last term in (8) and utilizing the geostrophic approximation for the wind and vorticity, together with the hydrostatic relationship, leads to

$$
\begin{aligned}
\frac{p f}{R} \overrightarrow{\partial p} \frac{\partial}{\partial p}(\mathbf{V} \eta) & =\frac{p f}{R} \frac{\partial \mathbf{V}}{\partial p} \cdot \nabla \eta+\frac{p f}{R} \mathbf{V} \cdot \nabla \frac{\partial \eta}{\partial p} \\
& =-J(T, \eta)-J\left(D, \underset{f}{g} \nabla^{2} T\right)
\end{aligned}
$$

Hence (8) becomes

$$
\begin{array}{r}
\sigma \nabla^{2} \omega+\frac{p f \eta}{R} \frac{\partial^{2} \omega}{\partial p^{2}}=\nabla^{2}\left[\frac{g}{-f} J(D, T)\right] \\
-J(T, \eta)-J\left(\frac{\left.\stackrel{g}{,} \underset{f}{-} \nabla^{2} T\right),}{}\right.
\end{array}
$$

where $D$ is the difference between the height of a pressure surface and the standard atmosphere value. This particular form has the advantage that no vertical derivatives appear in the "forcing function," i.e., the right side of (9), which may now be calculated from $D$ and $T$ values for each level.

\section{Boundary conditions}

Eq (9) is to be solved subject to certain boundary conditions. For the upper-boundary condition, $\omega$ is taken to be zero at $100 \mathrm{mb}$. Similarly $\omega$ vanishes at the lateral edges of the 1977-point octagonal grid, the latter being centered at the pole and extending to about 9 to $15 \mathrm{~N}$.

The lower-boundary condition is more involved and of particular interest in this investigation. In most previous studies the value of $\omega$ at the lower boundary has been calculated and applied at some low-level pressure surface usually $1000 \mathrm{mb}$. Here the lower-boundary vertical velocity, $\omega_{L 0}$, is computed and applied at terrain-height and is the resultant of the terrain-induced vertical velocity $\omega_{T}$ and the frictionally-induced vertical velocity $\omega_{F}$. The former may be expressed in the form

$$
\omega_{T}=\mathbf{V}_{T} \cdot \nabla p_{T}
$$

where $\boldsymbol{\nabla}$ is the two dimensional del-operator, $\mathbf{V}_{\boldsymbol{T}}$ is the horizontal wind and $p_{T}$, the pressure at terrain height.

In the derivation of the basic $\omega$-equation (6) friction has been neglected; however the divergence due to lowlevel friction can be taken into account via the lowerboundary condition (Sawyer, 1959). The form used here is similar to Cressman (1960)

$$
\omega_{F}=\frac{g_{\rho_{T}}}{f}\left[\frac{\partial}{\partial y}\left(C_{D} u_{T} V_{T}\right)-\stackrel{\partial}{\partial x}\left(C_{D} v_{T} V_{T}\right)\right],
$$

where $C_{D}$ is the drag coefficient.

In the initial calculations the variation of $C_{D} V_{T}$ was neglected, in which case (11) reduces to a somewhat more familiar form

$$
\omega_{F}=-\frac{g p_{T} C_{D} V ! n \zeta_{T}}{f R T_{T}}
$$

However, the complete form (1L) was used in some later computations.

\section{Finite difference equations}

The conventional centered finite difference analogs were used to approximate all horizontal derivatives. However to utilize directly the co nputer-analyzed temperature and pressure-height da:a at 1000, 850, 700, 500 , and $300 \mathrm{mb}$, non-centered finite difference analogs were necessary to approximate the vertical derivatives. These analogs were based on civerlapping parabolas fitted to each set of three successive pressure levels beginning at the terrain-pressure, $p_{T}$, and extending to $100 \mathrm{mb}$. For the first and second derivatives with respect to $p$, the finite difference formulas, are respectively

$$
\begin{aligned}
& \left(\frac{\partial A}{\partial p}\right)_{K}=\frac{1}{\Delta p_{U L}}\left[\frac{\Delta p_{U}}{\Delta p_{L}} A_{L}\right. \\
& \left.\quad-\left(\frac{\Delta p_{U}}{\Delta p_{L}}-\frac{\Delta p_{L}^{\prime}}{\Delta p_{U}}\right) A_{K}-\frac{\Delta p_{L}}{\Delta p_{U}} A_{U}\right], \\
& \left(\frac{\partial^{2} A}{\partial p^{2}}\right)_{K}=\frac{2}{\Delta p_{U} \Delta p_{U L}}-\left[A_{U}-\frac{\Delta p_{U L}}{\Delta p_{L}} l_{K}+\frac{\Delta p_{U}}{\Delta p_{L}} A_{L}\right],
\end{aligned}
$$

where $\Delta p_{U}$ is the difference belween the pressure at level $K$ and the pressure at the level above; $\Delta p_{L}$, the difference between the pressure it the level below and at $K$; and $\Delta p_{U L} \equiv \Delta p_{L}+\Delta p_{U}$. Th. ese expressions reduce the familiar centered difference analogs when $\Delta p_{U}=\Delta p_{L}$. To approximate the second derivative of $\omega$ in (9), (14) is to be used; and for $\sigma$ given by (3), (13) is appropriate. $\mathrm{Eq}(9)$ is to be solved numerically at the $850-, 700-$, $500-$ and $300-\mathrm{mb}$ levels by treating it as a system of Helmholtz-type equations for these levels. For this purpose it is necessary for convargence to control the stability parameter so that it dors not become negative or zero, the latter condition corresponding to a dryadiabatic lapse rate. While such a lapse rate is unlikely over the deep layers under consideration, nevertheless, precaution was taken in the prigram to prevent such an occurrence. Specifically, $\sigma$, which is equivalent to the 
difference between the dry-adiabatic lapse rate $\gamma_{p d}$ in pressure coordinates and the lapse rate, $\gamma_{p}=\partial T / \partial p$,

$$
\sigma=\gamma_{p d}-\gamma_{p}
$$

was not allowed to become less than one eighth the dryadiabatic value. Thus

$$
\sigma \geq \gamma_{p d} / 8
$$

Comparison of the actual $\sigma$ with the limiting value given in (16) for several synoptic cases showed that the criterion (16) is very rarely, if ever, invoked when the input data is hydrostatically consistent. '

In order to calculate $\sigma$ at $300 \mathrm{mb}$ according to (3) and (13), the temperature at some higher level is needed. Computer-analyzed data were not available at altitudes above $300 \mathrm{mb}$, hence a 100 -mb temperature field was determined from climatological data. Since the synoptic case chosen for study was in January 1962, 5-year mean 100-mb temperatures for January (1949-1953) were used. While this arrangement is not entirely satisfactory, the influence of the climatological data on the resulting vertical velocity field at $300 \mathrm{mb}$ is slight. The computer program is so arranged that synoptic analyses of the $100-\mathrm{mb}$ temperature field can be used when available on an operational basis.

Use of the finite difference analogs for the derivatives in (9) leads to an equation of the form

$$
\begin{array}{r}
\nabla^{2} \omega_{K}-B_{K} \omega_{K}=C_{K}+C_{\omega K} \\
K=1,2,3,4,
\end{array}
$$

where $\nabla^{2}$ represents the finite difference operator, the indices $K=1,2,3,4$ designate the pressure levels from $300 \mathrm{mb}$ to $850 \mathrm{mb}$ and the symbol $C_{\omega K}$ denotes the term involving the $\omega$ 's at levels above and below the level in question.

\section{Method of solution}

Eq (17) was solved by a three-dimensional accelerated Liebmann relaxation scheme, where the $n+1$ iterate, designated by a superscript, for any point is given by

$$
\omega^{n+1}=\omega^{n}+\frac{\lambda R^{n}}{4+B}
$$

Here $\lambda$ is the over-relaxation coefficient and the residual $R^{n}$ at any step is, as usual,

$$
R^{n}=\bar{V}^{2} \omega^{n}-B \omega^{n}-C-C_{\omega}{ }^{n} .
$$

According to a paper by 'Arnason (1956), certain conditions on the coefficients of (17) must be fulfilled for convergence as follows:

(a) $B_{K}>0$;

(b) Except for the minus sign, the sum of the coefficients of $\omega_{K-1}$ and $\omega_{K+1}$ must be less than or equal to the coefficient $B_{K}$, i.e.,

$$
B_{K}\left[\frac{\Delta p_{L}}{\Delta p_{U L}}+\frac{\Delta p_{U}}{\Delta p_{U L}}\right] \leq B_{K}
$$

The latter condition is automatically fulfilled except for possible round-off errors which were provided for within the program.

A remark should be made here concerning the treatment of the internal boundaries. Rather than simply placing $\omega$ equal to zero at grid points on a pressure surface "within the terrain," the computed vertical velocity $\omega_{L 0}$ for the lower boundary $p_{T}$ (at a lower pressure than the pressure surface) was entered at the grid point and held constant during the relaxation procedure. It was felt that this would result in more realistic values for $\nabla^{2} \omega$ at points adjacent to these internal boundary points.

The four pressure levels were successively scanned, beginning at $850 \mathrm{mb}$ and ending at $300 \mathrm{mb}$. Each pass over the four levels covered $4 \times 1977=7908$ points in the three-dimensional mesh and will be referred to as one SCAN.

The convergence criterion is simply that iteration ceases when

$$
\omega^{n+1}-\omega^{n}<\epsilon
$$

for all points of the mesh. Here $\epsilon$ is a predetermined quantity chosen so that no meteorologically significant differences exist between $\omega^{n+1}$ and $\omega^{n}$. The smallest value of $\omega$ considered to be of interest here was $10^{-4} \mathrm{mb}$ $\mathrm{sec}^{-1}$, which is approximately equal to $0.1 \mathrm{~cm} \mathrm{sec}^{-1}$ at $850 \mathrm{mb}$. A number of different values of $\epsilon$, ranging from $10^{-4}$ to $\left(10^{-4} / 6400\right) \mathrm{mb} \mathrm{sec}^{-1}$, were tried to determine a suitable value. The final value adopted was $\epsilon=\left(10^{-4} / 25\right)$ $\mathrm{mb} \mathrm{sec}^{-1}$. With this value convergence is reached in the case studied in 14 to 15 SCAN's over the three-dimensional mesh. On the other hand, a value of $\epsilon=\left(10^{-4} / 100\right)$ $\mathrm{mb} \mathrm{sec}^{-1}$ required about 26 SCAN's for convergence.

The Helmholtz-type of relaxation used here appears to converge more rapidly than the scheme used by Stuart ${ }^{3}$ and others, which omits the variable $B$ appearing in (18).

The over-relaxation coefficient $\lambda$ was determined experimentally so as to require the minimum number of SCAN's for a given $\epsilon$. The optimum $\lambda$ is very sharply defined and was found to be 1.414. A value differing from this by 10 per cent increased the SCAN count by about 25 per cent, while a change in $\lambda$ of only 0.1 per cent could change the SCAN count by 1 .

A final comment in connection with convergence concerns the initial guess field for the $\omega$ 's. Since in general there was a non-zero value at the lower boundary, it was thought that an initial guess of $\omega$ based on $\omega_{L 0}$ would facilitate convergence. It was expected that the influence of the lower boundary on the $\omega$-field would

${ }^{3}$ Stuart, D. W., 1961: Vertical motion and the baroclinic mechanism of rapid upper level cyclogenesis. Dept. of Meteor. Univ. of Calif., Los Angeles, $228 \mathrm{pp}$. 


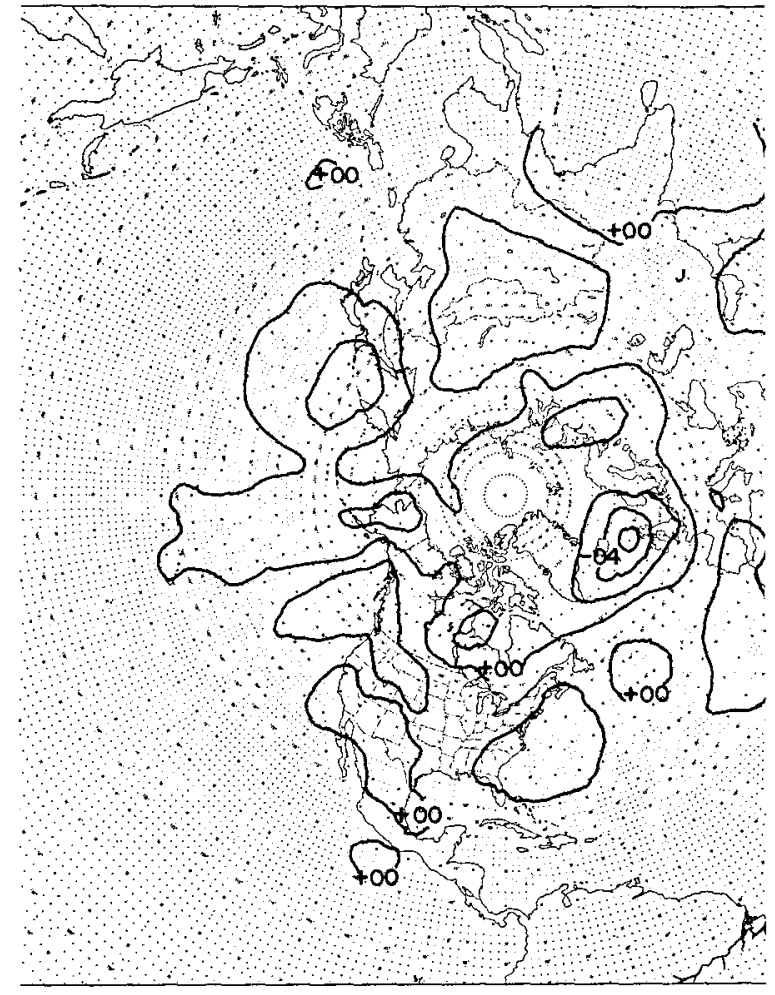

FIG. 1. 1000-mb D field (100's of feet), 0000 GCT, 22 January 1962.

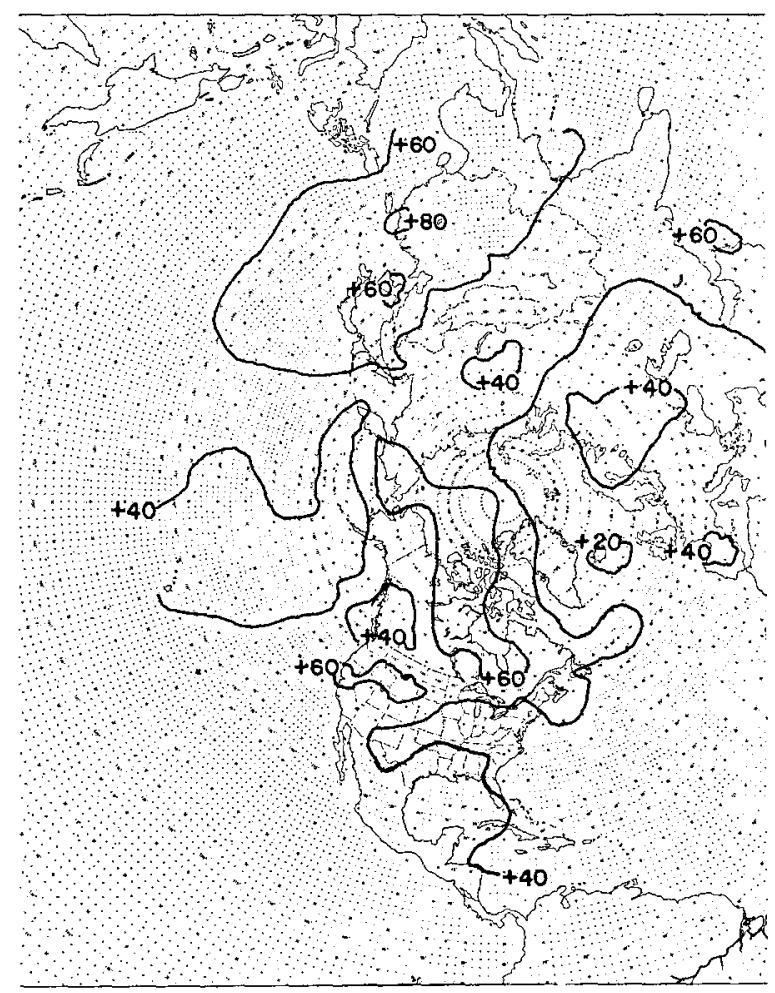

FIG. 3. 500-mb static stability (deg C per $1000 \mathrm{mb}$ ), 0000 GCT, 22 January 1962.

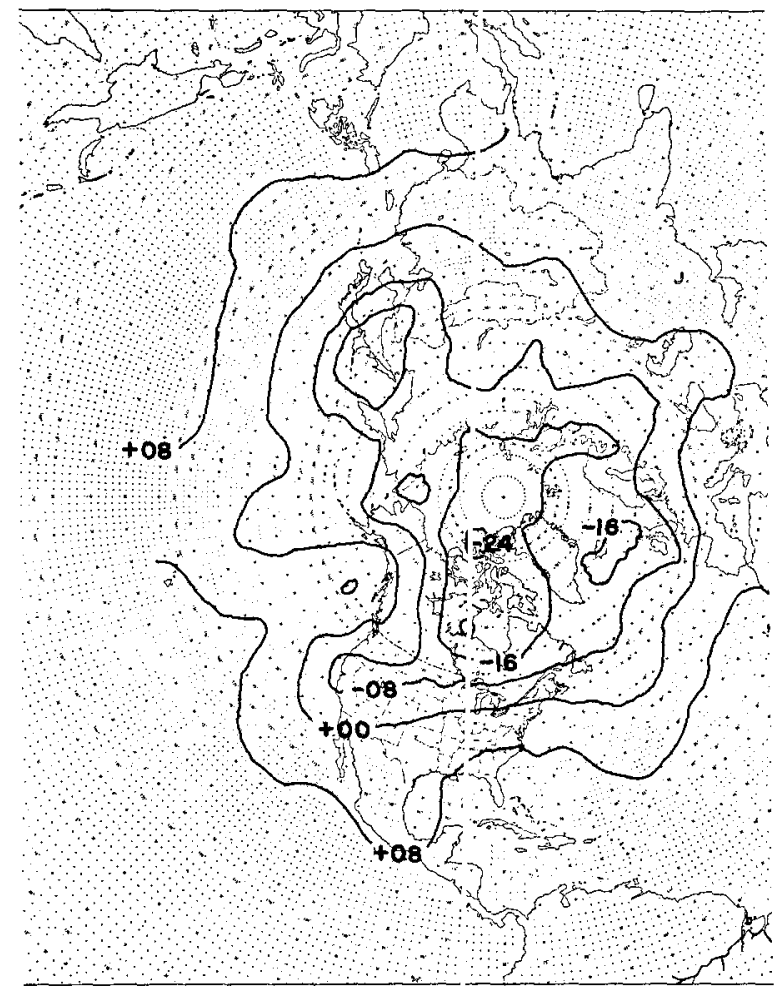

FIG. 2. 500-mb D field (100's of feet), 0000 GCT, 22 January 1562 .

decrease roughly at an exponential rate above the terrain, hence the initial guess field was taken as

$$
\omega_{K}^{0}=2^{-n} \omega_{L 0}
$$

with $n=1$ for the first pressure level above the terrain, $n=2$ for the second, etc. However, this procedure did not significantly facilitate convergence of the relaxation scheme as compared to an initic.l guess field of zero.

Embodied in the computer p.ogram is a "smoother" of the form

$$
\widetilde{A}=A+r \nabla^{\prime}: A,
$$

where $r$ is a constant which can be varied. The purpose of this operator is to control sinall scale irregularities, particularly in the fields invo ving second and third order differences, namely,

$$
\nabla^{2} D, \nabla^{2} T \text { and } \nabla^{2} J(D, T) .
$$

A value of $r=\frac{1}{8}$ was used in thes a particular experiments. As might be expected this pocedure removes some minor irregularities as well as reducing peak values in the $\omega$-fields. The initial basis for introducing this smoother was the appearance of some "choppiness" in the vorticity fields.

To perform the computations necessary for this investigation, a Control Data Corporation (CDC) 1604 digital computer was utilized, frossessing a core memory of 32,768 words of 48 bits eac.1 and a typical add time 


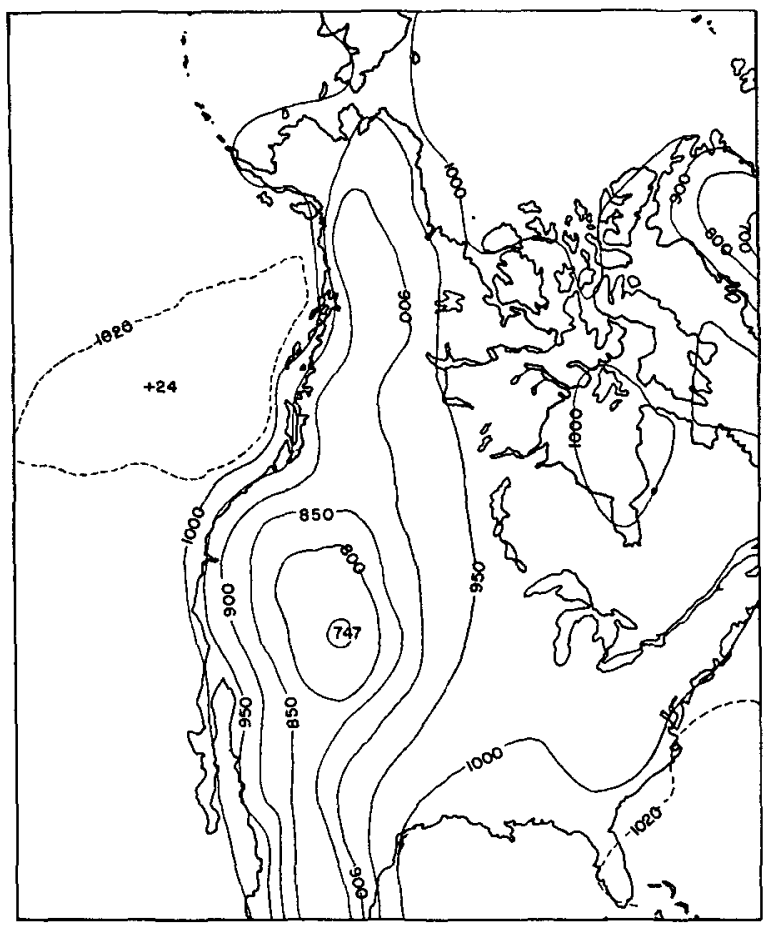

FIG. 4. Pressure at terrain height $p_{T}$ for $0000 \mathrm{GCT}$, 22 January 1962 .

of 7.2 microseconds. The time required for the entire program to compute the vertical velocity at the five levels is about three minutes.

\section{Results}

One of the purposes of this study was to investigate in somewhat greater detail than heretofore the influence of the lower boundary on the $\omega$-fields. Toward this end the computer program was designed:

a) to include or omit either the terrain-induced and/or the frictionally-induced vertical velocity at the lower boundary;

b) to apply the omega for the lower boundary $\omega_{L 0}$ at the pressure of the terrain height, or to apply it at a pressure based on the assumption that the lower boundary is at sea-level.

The last mentioned case is essentially the approximation normally used (see, for example, Stuart, footnote 3), wherein the lower-boundary value is arbitrarily assigned to the pressure level nearest the earth in the model, usually $1000 \mathrm{mb}$. In this event, the lower-boundary condition is not only being applied at the incorrect altitude, but also ignores internal boundaries where the terrain intersects a pressure surface.

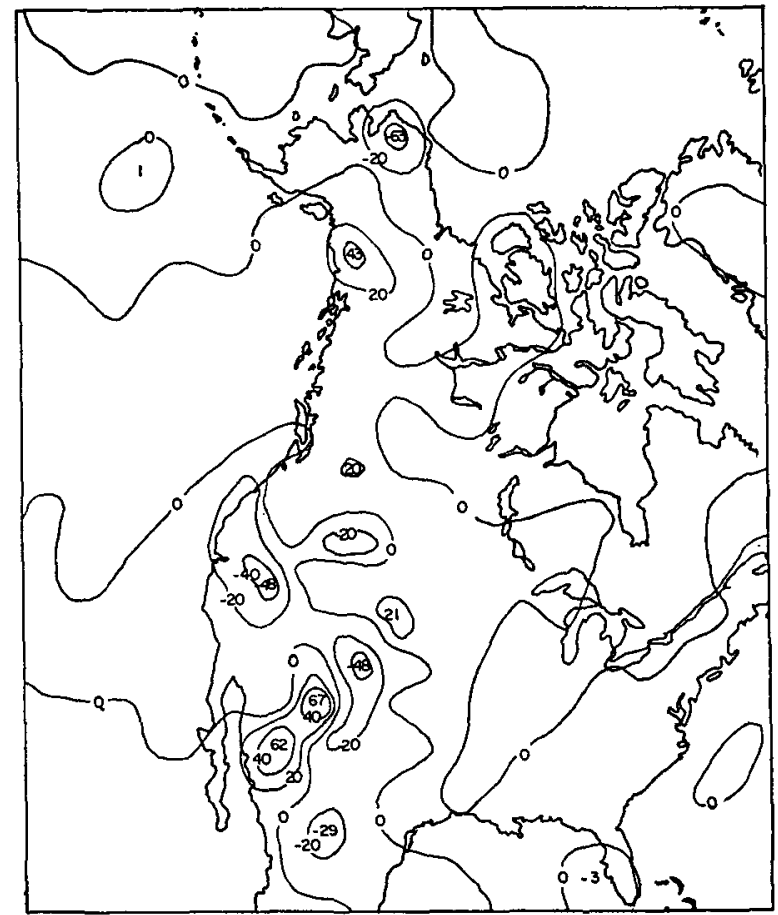

FIG. 5. Frictionally-induced vertical velocity $\omega_{F}$ at lower boundary in units of $10^{-4} \mathrm{mb} \mathrm{sec}^{-1}$ for $0000 \mathrm{GCT}, 22$ January 1962.

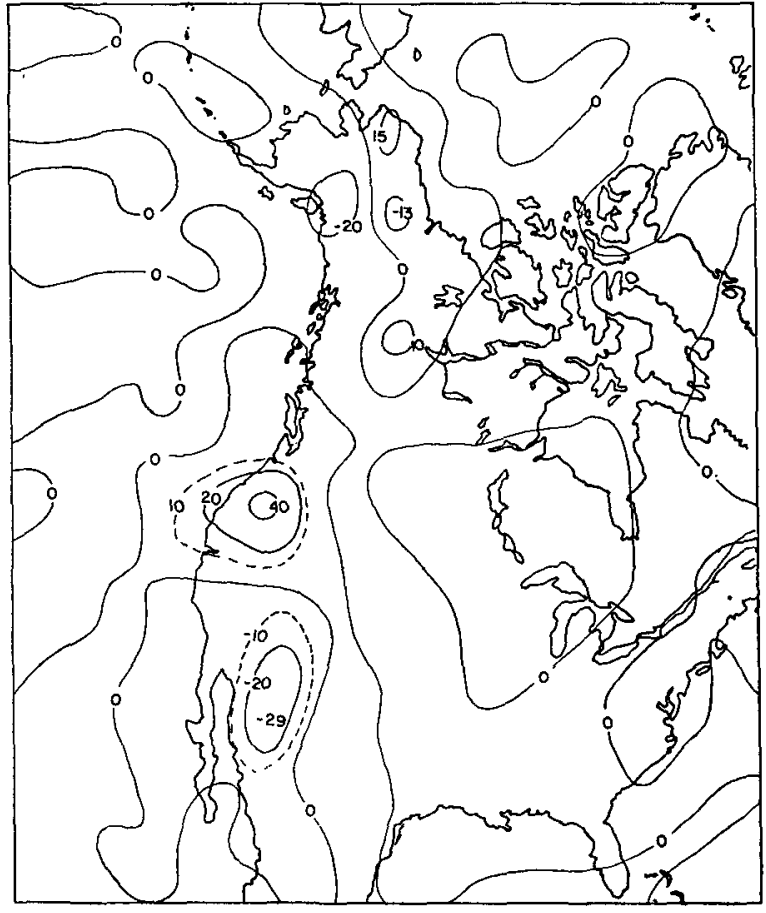

FIG. 6. Terrain-induced vertical velocity $\omega_{T}$ at lower boundary. 
To determine more precisely the vertical extent of the lower boundary influence, the forcing function, $C_{K}$ in (17), may be omitted. The solution then shows the decay of the terrain and friction influences with height, subject only to the variations of static stability.

Finally the effects of utilizing $f_{m}$ rather than $\eta$ in the divergence term of the vorticity equation and differences due to a constant $\sigma$ versus a variable $\sigma$ in (7) are considered.

The synoptic situation selected for study occurred in January 1962 when a severe storm brought heavy precipitation to the west coast of United States with considerable snow even to Southern California. Figs. 1 through 3 show the 1000 - and $500-\mathrm{mb} \mathrm{D}$ fields and the $500-\mathrm{mb} \sigma$ field for 0000 GCT 22 January. The $\omega$-fields will be given in units of $10^{-4} \mathrm{mb} \mathrm{sec}^{-1}$. In general, comparison of the $\omega$-fields for the various cases will be made to the case with a stability parameter which varies only with pressure, namely $\bar{\sigma}$, and also with the quantity $f_{M}{ }^{2}$ in the coefficient of $\partial^{2} \omega / \partial p^{2}$ (rather than $f \eta$ ).

\section{A. Lower boundary effects}

Fig. 5 shows $\omega_{F}$ as determined by $\mathrm{Eq}(12)$ at the variable lower boundary $p_{T}$. Quite large magnitudes of $\omega_{F}$ are found, the maximum in the area shown being -67 units of $10^{-4} \mathrm{mb} \mathrm{sec}^{-1}$. Moreover within a distance

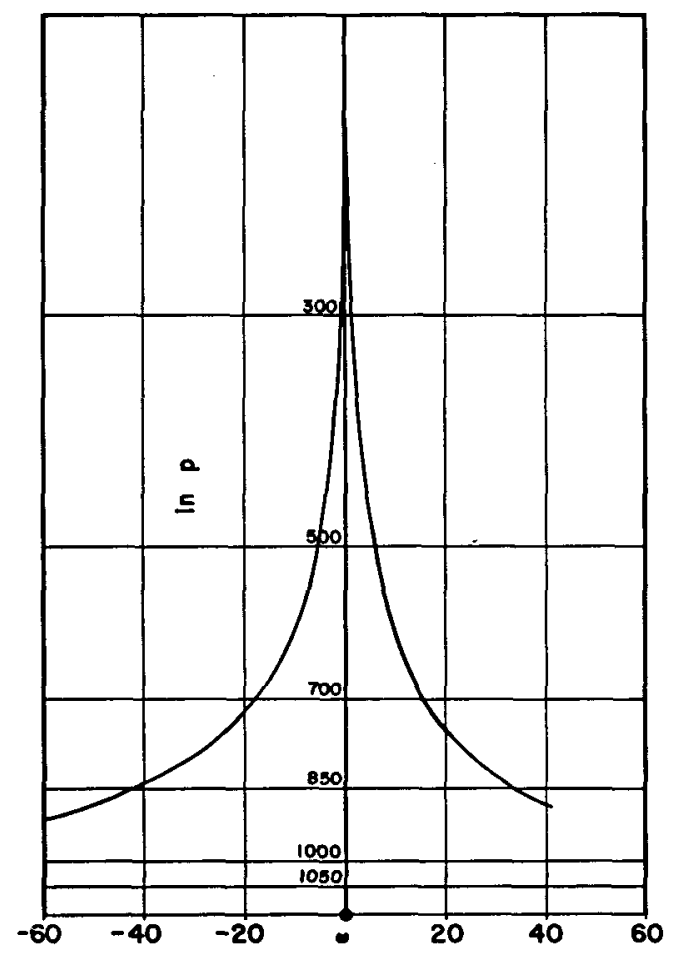

Fig. 7. Typical vertical profiles of $\omega_{F}$ (left) and $\omega_{T}$ (right) (units of $10^{-4} \mathrm{mb} \mathrm{sec}^{-1}$ ) versus $\ln p$ from surface maxima with forcing function zero. of a few hundred kilometers a positive value of +48 units appears. Values to several hundred units were found in a few places elsewhere. A.s may be seen by (12) the sign of $\omega_{F}$ is determined solely by the sign of the relative vorticity; however the magnitude is a function not only of $\zeta$ but also the wind speed and the drag coefficient. The drag coefficients, as given by Cressman (1960), are sometimes over six times greater in the mountainous terrain than over the ocean areas, which accounts to a large extent for the substantially larger values of $\omega_{F}$ over the mountainous areas. Nevertheless there appears to be somewhat excessive irregularity in the boundary values of $\omega$. Perhaps some smoothing of the surface vorticity field would better represent the large scale systems.

Fig. 6 gives the terrain-inducid vertical velocity as computed by (10). The values of $\omega_{T}$ are generally somewhat less in magnitude and show less detail than for $\omega_{F}$, at least for this particular synoptic situation. The maximum magnitude in the North American area is +40 units. Of course the terrain field is; greatly smoothed, the lowest pressure $p_{T}$ being only 74:7 mb over the Rocky Mountains (see Fig. 4), $698 \mathrm{ml}$ ) over Greenland and $522 \mathrm{mb}$ over the Himalayas. As : a matter of fact, larger values of $\omega_{T}$ were found over the Himalayas. Due to the heavy smoothing, terrain effects extend somewhat over adjacent ocean areas. In this particular situation downward motion is observed in the Oregon area due to offshore flow, whereas onshore flow gives rising currents in southwestern United States and Mexico.

$\mathrm{Eq}(17)$ was now solved with the forcing function $C_{K}$ identically zero. This was done separately with $\omega_{F}$ and $\omega_{T}$ as the lower-boundary condition. The decay of $\omega$ with height in these cases is illus,trated in Fig. 7 where the curve on the left is a typical rertical profile showing the frictionally-induced vertical velocity as a function of $\ln p$ at a surface maximum, 'while the curve on the right is a similar curve at a center of maximum terraininduced vertical velocity. The damping with height is somewhat less severe in the latter, perhaps because of the larger scale of the patterr.s of $\omega_{T}$ compared to those of $\omega_{F}$.

Figs. 8, 9 and 10 show the $u$-fields at the surface, 850 and $700 \mathrm{mb}$ with zero forcirg function $C_{K}$ and the combined frictionally-and terrain-induced velocities at the lower boundary. On the 850-rab chart the area where the terrain altitude exceeds that of the $850-\mathrm{mb}$ surface has been delineated. The maxim um $\omega$-value is 44 units in the western United States. The vertical velocity decreases rapidly with height with zero forcing function and by $500 \mathrm{mb}$ the maximum ragnitude found in the North American area is only 6 units.

Next some calculations wer: made for $\omega_{L 0}$ which included the variation of $C_{D} V_{T}$ i.e., $\omega_{F}$ was computed using the complete form (11) rather than the simplified 


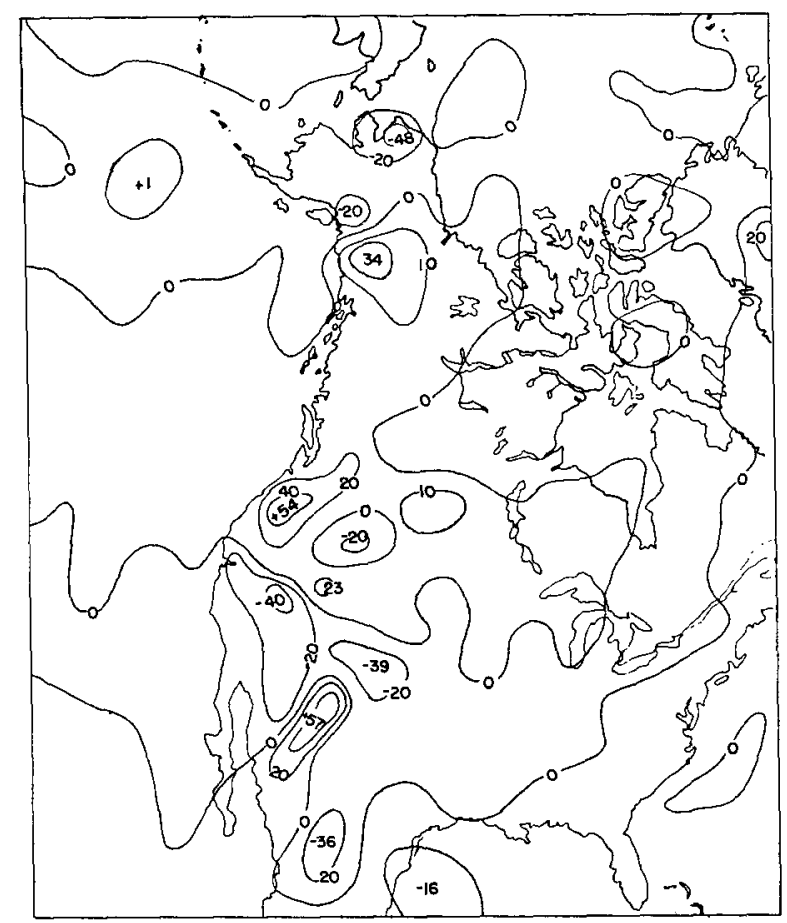

FIG. 8. Frictionally- and terrain-induced vertical velocity $\omega_{L O}$ at lower boundary for $0000 \mathrm{GCT}, 21$ January 1962.

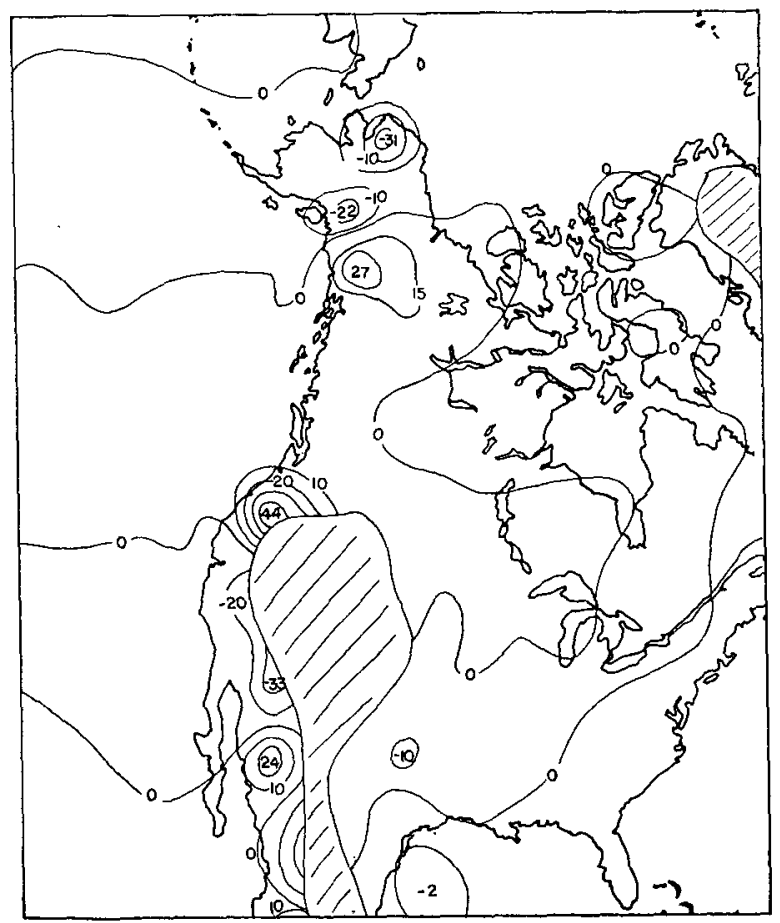

FIG. 9. Vertical velocity at $850 \mathrm{mb}$ with $\omega_{L O}$ at lower boundary $p$, and forcing function zero, for 0000 GCT, 21 January 1962. form (12). By and large the patterns of $\omega_{L 0}$ were similar in the two cases and the values generally of the same sign and approximate magnitude. With regard to the latter, the values of $\omega_{L 0}\left(10^{-4} \mathrm{mb} \mathrm{sec}^{-1}\right)$ at the important maxima and minima in the North American area are given in the following table with the first row including the complete form of $\omega_{F}\left(10^{-4} \mathrm{mb} \mathrm{sec}^{-1}\right)$ given by (11) and the corresponding figures in the second row for the approximate form (12). The average difference is about

$\begin{array}{lllllllllllll}48 & -32 & 47 & -23 & -48 & 27 & 34 & -39 & -35 & -32 & -28 & 34 & -44\end{array}$ $\begin{array}{lllllllllllll}57 & -44 & 54 & -23 & -48 & 34 & 29 & -36 & -19 & -23 & -27 & 29 & -44\end{array}$

6 units or about 15 to 20 per cent, which is not negligible; however the simpler form appears to give the essential features of the frictional influence at the lower boundary. Solutions of (17) showed that the differences due to the use of (11) versus (12) decreased with increasing altitude. At $850 \mathrm{mb}$ the maximum difference between the two cases was 11 units with an average difference of about 3 units at the centers of ascending and descending motion, while at $700 \mathrm{mb}$ the average difference at the centers was less than 2 units.

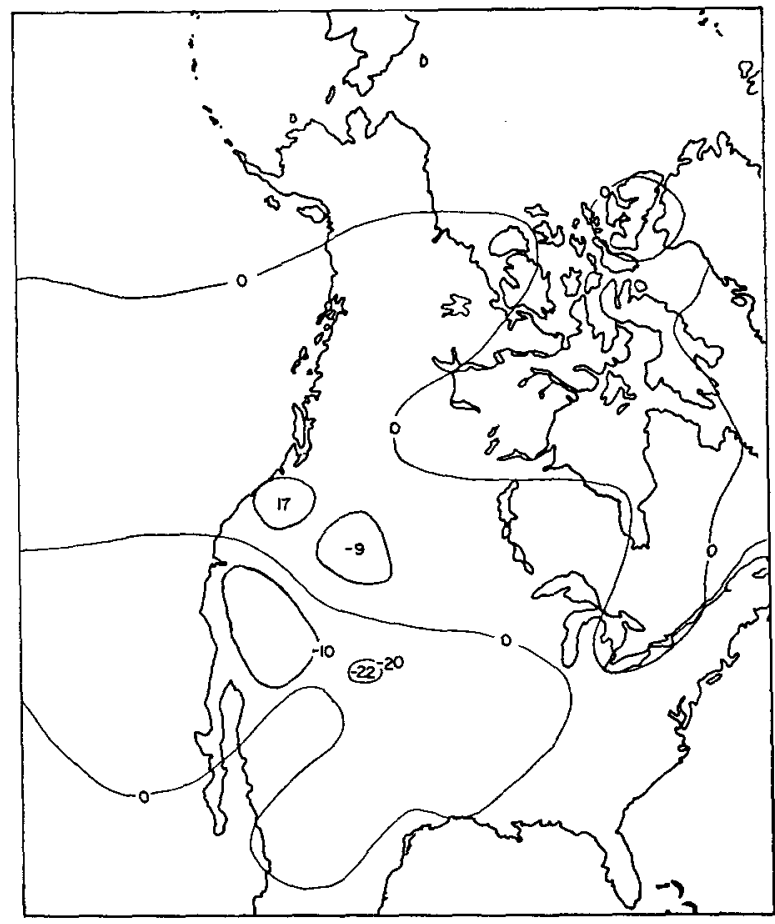

FIG. 10. Similar to Fig. 9.but for $700 \mathrm{mb}$. 


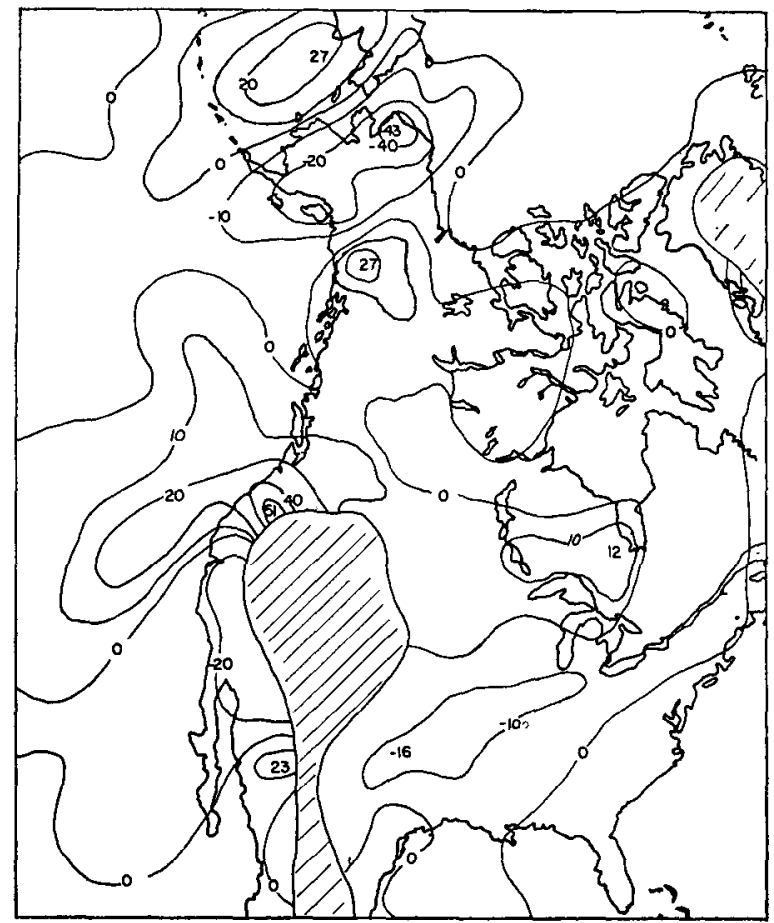

Fig. 11. Similar to Fig. 9 but with forcing function not zero, stability parameter $\bar{\sigma}$ varying with pressure only, and $f_{M^{2}}$ in coefficient of $\partial^{2} \omega / \partial p^{2}$.

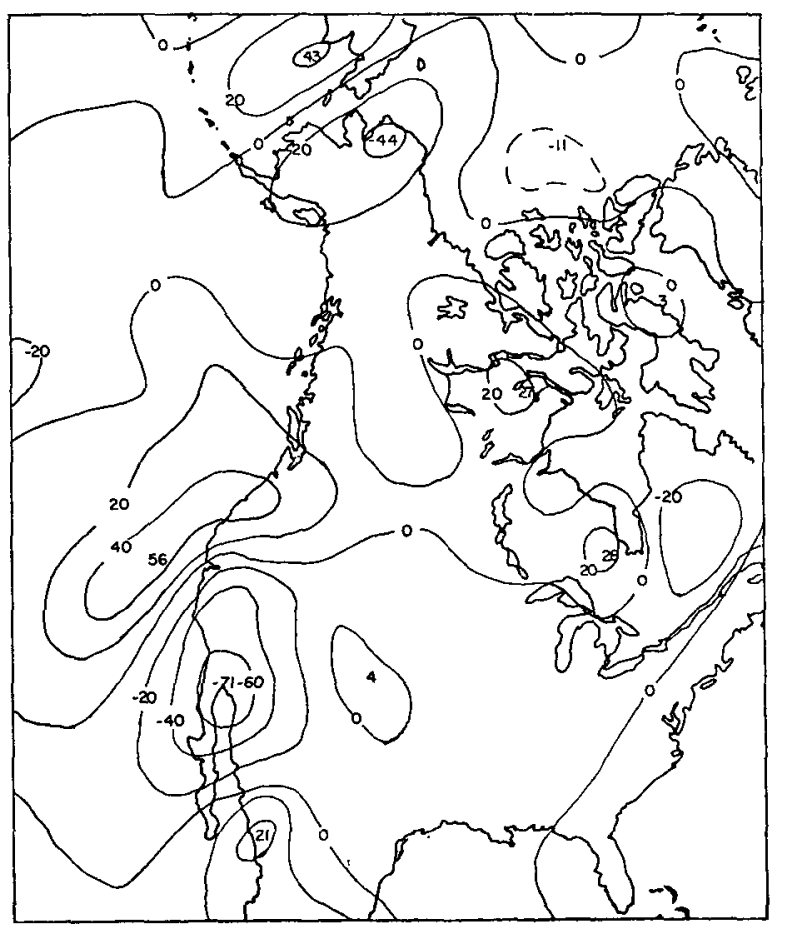

Fra. 13. Similar to Fig. 11 but for $500 \mathrm{mb}$.

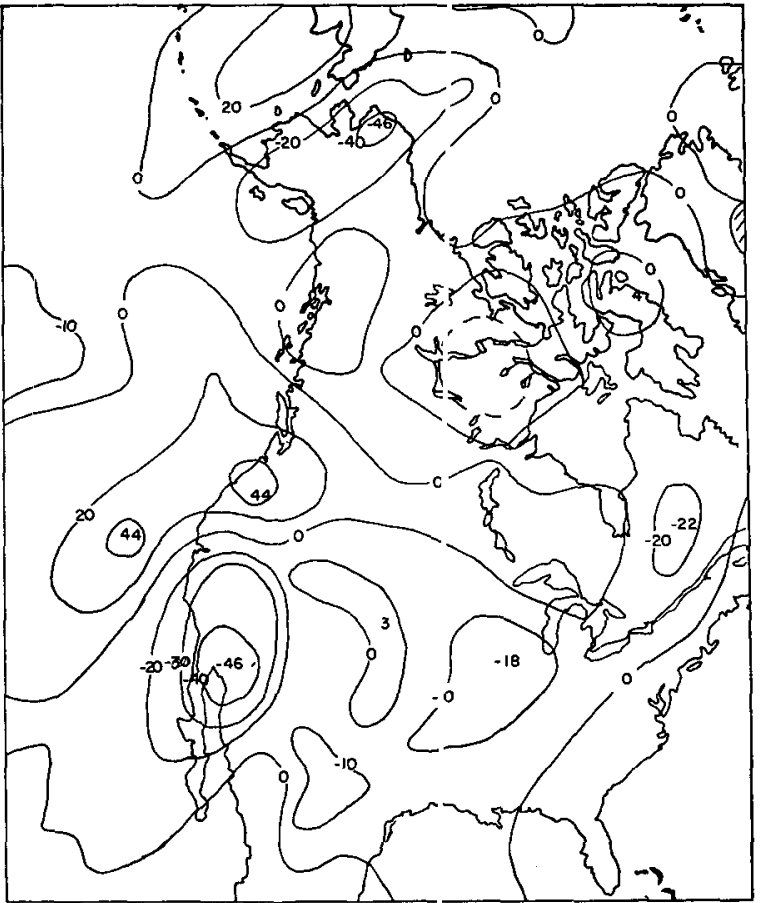

FIG. 12. Similar to Fig. 11 but for $700 \mathrm{mb}$.

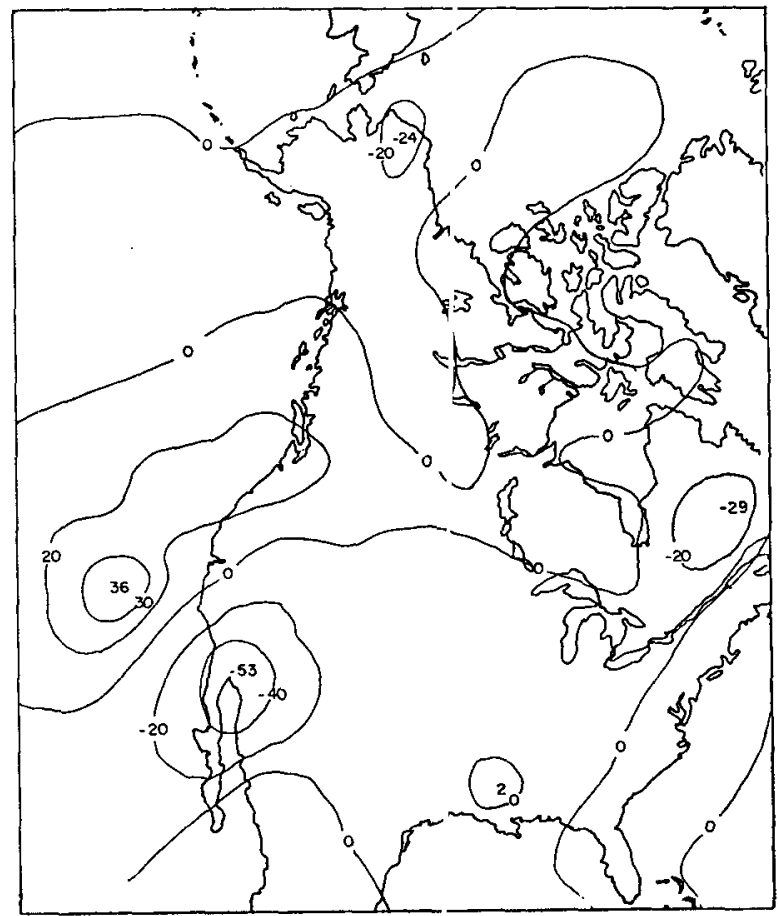

FIG. 14. Similar to Fig. 11 but for $300 \mathrm{mb}$. 


\section{B. "Standard" $\omega$-fields}

In order to ascertain the effects of the lower boundary, varying static stability, etc., some calculations were first made with the following "standard" conditions:

1) frictionally-and terrain-induced velocities at the lower boundary, as given by Fig. 8;

2) lower-boundary condition applied at terrain pressure;

3) static stability parameter a function of pressure only;

4) the coefficient $f_{M}{ }^{2}$ (rather than $f \eta$ ) for the second derivative $\partial^{2} \omega / \partial p^{2}$.

Figs. 11, 12, 13 and 14 give the $\omega$-fields at $850,700,500$, and $300 \mathrm{mb}$ for the above "standard" conditions. The forcing function is now present and substantial values of $\omega$ are found at 500 and $300 \mathrm{mb}$. The static stability $\bar{\sigma}$, in units of deg $\mathrm{K}$ per $100 \mathrm{mb}$, had the following values:

$$
\begin{array}{lcccc}
p(\mathrm{mb}) & 850 & 700 & 500 & 300 \\
\tilde{\sigma} & 5.07 & 5.29 & 4.80 & 10.17
\end{array}
$$

Fig. 15 gives several typical vertical profiles of $\omega$ versus $\ln p$ taken from places of either surface or midtroposphere maximum vertical velocity. While many of the profiles were simple in form such as the one on the right, others were somewhat complicated as these examples show.

The next computations made were similar to those above except that the lower-boundary condition was applied at a sea-level pressure determined by assuming the terrain-height to be zero everywhere. Thus the pressure at the lower boundary differs only insignificantly from $1000 \mathrm{mb}$; however the friction and mountain influences with respect to $\omega_{L 0}$ are fully included. In this case fictitious vertical velocities appear in areas where $p_{T}<850 \mathrm{mb}$; and where $p_{T}$ is actually considerably less than $1000 \mathrm{mb}$, the influence of $\omega_{L 0}$ is falsely reduced to a large degree. Differences of 20 to 30 units occur at 850 $\mathrm{mb}$ giving errors as much as 100 per cent. Thus the application of the lower-boundary condition incorrectly at sea level gives quite an erroneous picture of the vertical velocity field at $850 \mathrm{mb}$ over mountainous areas. By $700 \mathrm{mb}$, however, with this rather heavily-smoothed terrain, the differences resulting from applying the lower-boundary value at the sea-level pressure rather than at $p_{T}$ are generally rather minor over the North American area, and by $500 \mathrm{mb}$ have disappeared altogether. On the other hand, over the Himalaya Mountains, the effects were still significant at $700 \mathrm{mb}$ and even to some extent at $500 \mathrm{mb}$.

Still another computation was made with the lower condition of $\omega=0$. Marked differences occur, especially in the mountainous areas characterizing the entire western coast of North America. These results reaffirmed the necessity of including mountain and friction influences if a reasonable picture of even the large-scale vertical velocity field in the lower troposphere is to be obtained.

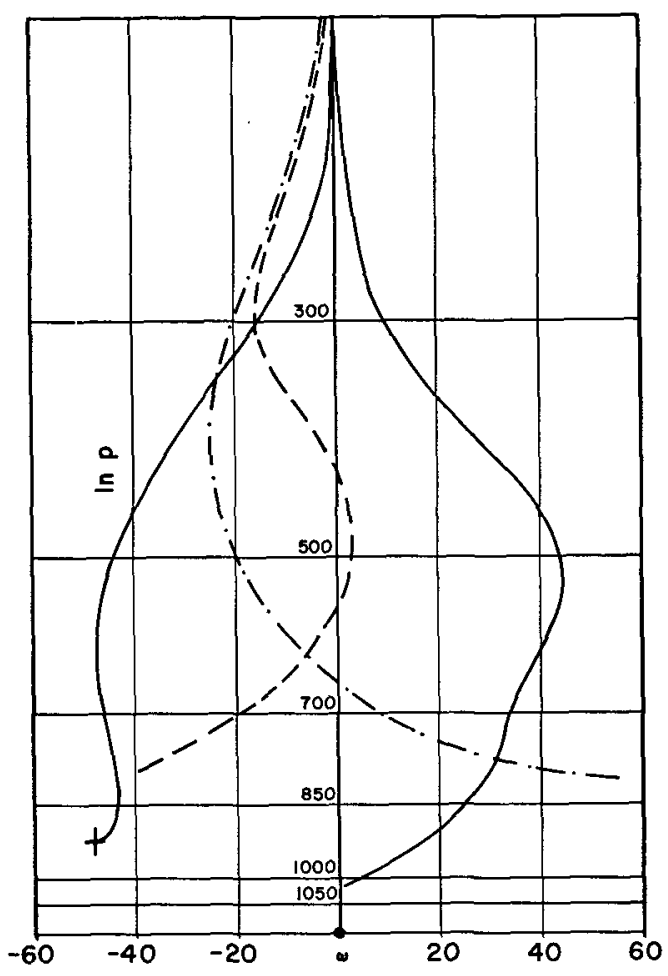

FIG. 15. Some typical profiles of $\omega$ (units of $10^{-4} \mathrm{mb} \mathrm{sec}^{-1}$ ) versus $\ln p$ associated with either surface or $500-\mathrm{mb}$ maxima.

\section{The effect of variable static stability}

Considered next is the influence of a variable static stability, as governed by the parameter $\sigma$, on the vertical velocity field. This was accomplished by a series of calculations which systematically utilized 1) an over-all mean static stability $\overline{\bar{\sigma}}$, which had a value of $6.33 \mathrm{deg} \mathrm{K}$ per $100 \mathrm{mb}$ for this case; 2 ) a $\bar{\sigma}$ which varied only vertically in $(8) ; 3)$ a point-variable static stability $\sigma$ in $\mathrm{Eq}$ $(8)$; 4) a comparison of the magnitude of the three terms comprising $\nabla^{2}(\sigma \omega)$ as given by (7); and finally 5) the addition of the term $\omega \nabla^{2} \sigma$ to the left member of (8).

The results of the first three series of computations listed above are partially indicated by the following tabulation which gives the negative $\omega$ values of maximum magnitude associated with the low pressure centered in southwestern United States and the maximum positive values just to the northwest. The negative values at 700 and $500 \mathrm{mb}$ in the $\overline{\bar{\sigma}}$ case are numerically smaller than in the other cases, due apparently to the slightly greater stability imposed by the over-all aver-

\begin{tabular}{ccccccc}
\hline & \multicolumn{3}{c}{ Negative centers } & \multicolumn{3}{c}{ Positive centers } \\
& 700 & 500 & 300 & 700 & 500 & 300 \\
\hline$\sigma$ & -50 & -70 & -51 & 44 & 58 & 38 \\
$\bar{\sigma}$ & -46 & -71 & -53 & 44 & 56 & 36 \\
$\overline{\bar{\sigma}}$ & -43 & -68 & -67 & 41 & 51 & 46 \\
\hline
\end{tabular}


age. However the $\omega$ value at $300 \mathrm{mb}$ in the $\overline{\bar{\sigma}}$ case is substantially greater than the other cases because both $\sigma$ and $\bar{\sigma}$ are much more stable than $\overline{\bar{\sigma}}$ at $300 \mathrm{mb}$, tending to suppress vertical motion. The stability parameter computations, particularly for $700 \mathrm{mb}$, showed southwestern United States to be an area of relatively low static stability on this date. The differences between the $\omega$ values with $\bar{\sigma}$ and $\sigma$ are rather slight except perhaps at $700 \mathrm{mb}$, where about an 8 per cent difference occurs in the upward motions.

With regard to the positive values of $\omega$, the slightly smaller magnitudes in the $\overline{\bar{\sigma}}$ case at 700 and $500 \mathrm{mb}$ may be due to the stabilizing influence of $\overline{\bar{\sigma}}$ compared to $\sigma$ or $\bar{\sigma}$. The largest differences occur again at $300 \mathrm{mb}$ where vertical velocities appear to be exaggerated in the $\overline{\bar{\sigma}}$ case because of the insufficient static stability at $300 \mathrm{mb}$ when an over-all mean value is used.

In the Alaskan area the following positive and negative $\omega$ values were observed in the three cases. Here

\begin{tabular}{cccccccc}
\hline & \multicolumn{3}{c}{ Positive centers } & \multicolumn{3}{c}{ Negative centers } \\
& 700 & 500 & 300 & 700 & 500 & 300 \\
\hline$\sigma$ & 28 & 36 & 11 & -44 & -41 & -19 \\
$\bar{\sigma}$ & 35 & 43 & 18 & -46 & -44 & -24 \\
$\overline{\tilde{\sigma}}$ & 31 & 38 & 23 & -42 & -41 & -29 \\
\hline
\end{tabular}

again the $\omega$ values at $300 \mathrm{mb}$ are exaggerated in the $\overline{\bar{\sigma}}$ case, even more so percentagewise than in the United States area. At 700 and $500 \mathrm{mb}$ the differences are rather small in the center of ascending motion; however, rather substantial differences occur with the descending motion $(+\omega)$ at these levels.

The next step in the evaluation of the influence of static stability on the vertical velocity field consisted of comparing the magnitudes of the three terms comprising the right hand member of (7), the purpose being to ascertain if the inclusion of the additional terms $\omega \nabla^{2} \sigma$ and $2 \nabla \sigma \cdot \nabla \omega$ would significantly modify the computed vertical velocities.

Computations were made for 1200 GCT, 22 January 1962 , during the storm period. The term $\sigma \nabla^{2} \omega$ had a magnitude exceeding 10 arbitrary units at 820 of a possible 1709 points (fixed lateral boundary points were excluded in the count), and the maximum value was 738 units.

On the other hand, the term $\omega \nabla^{2} \sigma$ had a magnitude in excess of 10 of the arbitrary units at 68 of the 1709 points with a maximum value of 140 units. Finally, the magnitude of the term $2 \nabla \omega \cdot \nabla \sigma$ exceeded 10 units at only 25 of 1709 points with a maximum value of 92 units.

Thus in this synoptic situation, the mean value of $\sigma \nabla^{2} \omega$ was one to two orders of magnitude greater than either of the other two terms.

Finally some computations of $\omega$ were made with the two terms, $\sigma \nabla^{2} \omega+\omega \nabla^{2} \sigma$, included in the $\omega$-equation. This was done because the addition of the new term $\omega \nabla^{2} \sigma$ to the previous program for solvin $\mathrm{Eq}^{\mathrm{E}}(8)$ was relatively simple, while the inclusion of $2 \nabla \omega \cdot \nabla \sigma$ would have required somewhat more extensive programming. Moreover the previously mentioned computations had shown that $2 \nabla \omega \cdot \nabla \sigma$ was the least impos tant of the three terms of (7). The results which are for $0000 \mathrm{GCT}, 22$ January 1962 , were then compared to th sse obtained previously with only $\sigma \nabla^{2} \omega$. As might now be expected from the foregoing comparison of these terms, the differences were slight indeed. Table 1 giv ss the magnitudes of $\omega$

Table 1. Values of $\omega\left(10^{-4} \mathrm{mb} \mathrm{sec}^{-1}\right)$ computed without (Case 1) and with (Case 2) the term $\omega \nabla^{2} \sigma$.

\begin{tabular}{lcccc}
\hline & $850 \mathrm{mb}$ & $700 \mathrm{r} \mathrm{b}$ & $500 \mathrm{mb}$ & $300 \mathrm{mb}$ \\
\hline $\begin{array}{l}\text { Alaskan } \\
\text { area }\end{array}$ & Case 1 $+22,-43+28,-44+35,-42+11,-19$ \\
& Case 2 $+22,-43+28,-44+36,-41+11,-18$ \\
$\begin{array}{l}\text { Western } \\
\begin{array}{l}\text { United } \\
\text { States }\end{array}\end{array}$ & Case 1 $+45,-35$ & $43,-51+52,-67+28,-40$ \\
\end{tabular}

at the centers of ascending and descending motion without $\omega \nabla^{2} \sigma$ (Case 1) and with $\omega \nabla^{2} \sigma$ (Case 2) included in the $\omega$-equation. It is appareat that the inclusion of this term made little difference in vertical velocity fields and this was true not only at the centers of vertical motion but everywhere over th 2 grid.

Since the term $2 \nabla \omega \cdot \nabla \sigma$ was of even smaller magnitude than $\omega \nabla^{2} \sigma$, it may be reasonakily inferred that its influence on the $\omega$-fields would certainly be no greater. Thus in this example the term $\nabla^{2}(\sigma \omega)$ is approximated adequately by the expression $\sigma \nabla^{2} \omega$ with respect to the computation of large-scale vertical velocity.

It should be emphasized that these conclusions are based mainly on a particular synoptic situation; however their reliability is enhanced by hemispheric extent of the computations.

Another point to be considered is the grid distance of $381 \mathrm{~km}$, which certainly does not permit an estimation of phenomena of short wavelength, say less than roughly $1000 \mathrm{~km}$; nor does it reflect the intense gradients of meteorological parameters in the vicinity of fronts, squall lines and mesoscale pher.omenon in general.

\section{Effect of $f \eta$ versus $f_{M}{ }^{2}$}

This section considers the effect of replacing the coefficient $f \eta$ of the second deriva ive $\partial^{2} \omega / \partial p^{2}$ in (8) by the approximate form $f_{M^{2}}$. Firstly it should be mentioned that the number of SCAN's rec uuired for convergence in the $f \eta$ case was half again as great as for the $f_{M}{ }^{2}$ case, increasing the time for solution considerably. The tabulation below gives the $\omega$ values; at the centers of maximum upward and downward motion along the west coast of the United States and also in the Alaskan area. 


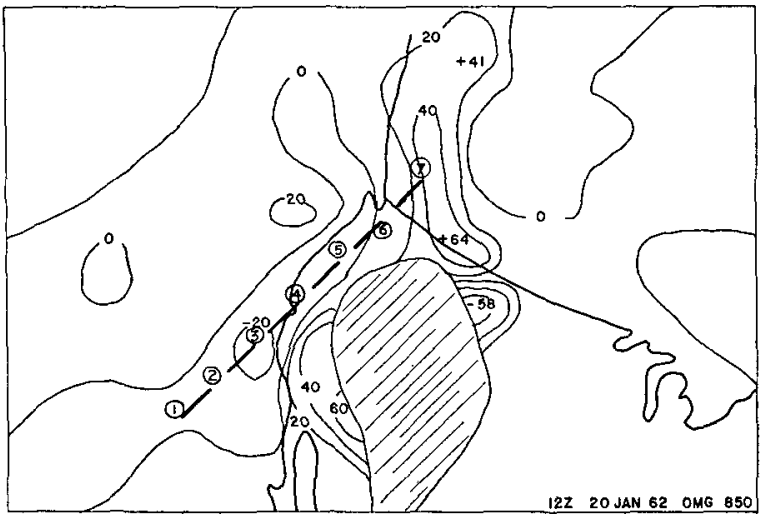

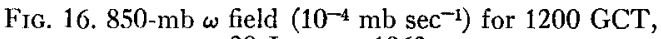
20 January 1962.

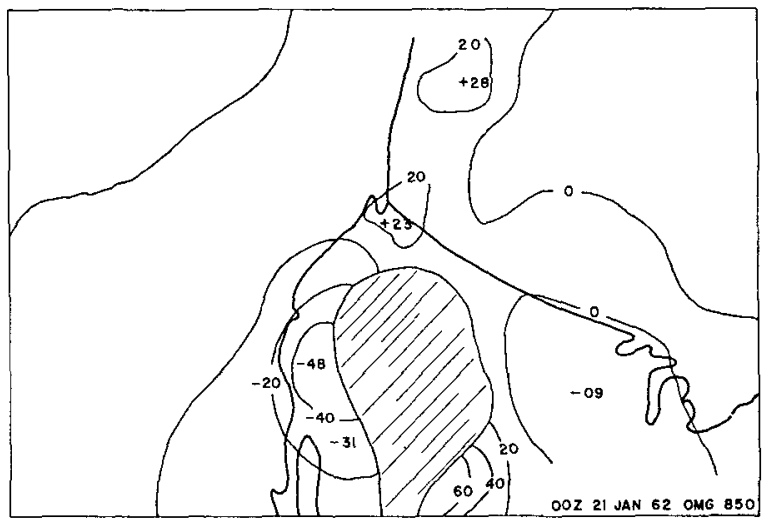

FIG. 18. $850-\mathrm{mb} \omega$ field $\left(10^{-4} \mathrm{mb} \mathrm{sec}^{-1}\right)$ for $0000 \mathrm{GCT}$, 21 January 1962.

\begin{tabular}{lrrrrrr}
\hline & \multicolumn{8}{c}{ Western United States } \\
& \multicolumn{7}{c}{ Positive center } & \multicolumn{2}{c}{ Negative center } \\
& 700 & 500 & 300 & 700 & 500 & 300 \\
\hline$f_{M}{ }^{2}$ & 44 & 56 & 36 & -46 & -71 & -53 \\
\hline$\eta$ & 46 & 57 & 41 & -50 & -77 & -56 \\
\hline & 700 & 500 & 300 & 700 & 500 & 300 \\
\hline$f_{M}{ }^{2}$ & 35 & 43 & 18 & -46 & -44 & -24 \\
\hline$f_{\eta}$ & +27 & +32 & +15 & -42 & -37 & -21 \\
\hline
\end{tabular}

In the western United States the magnitudes of the $\omega$ 's in $f \eta$ case are slightly larger than for the $f_{M}{ }^{2}$ case by 1 to 6 units, amounting to a maximum difference of roughly 15 per cent. On the other hand, in the Alaskan area the magnitudes of the $\omega$ 's in the $f \eta$ case are systematically smaller than in the $f_{M}{ }^{2}$ case by 3 to 11 units, amounting to a maximum difference of approximately 25 per cent. Examination of other areas of the Northern Hemisphere showed the differences of $\omega$ between the $f_{M}{ }^{2}$ and $f \eta$ cases to be usually rather small, but occa-

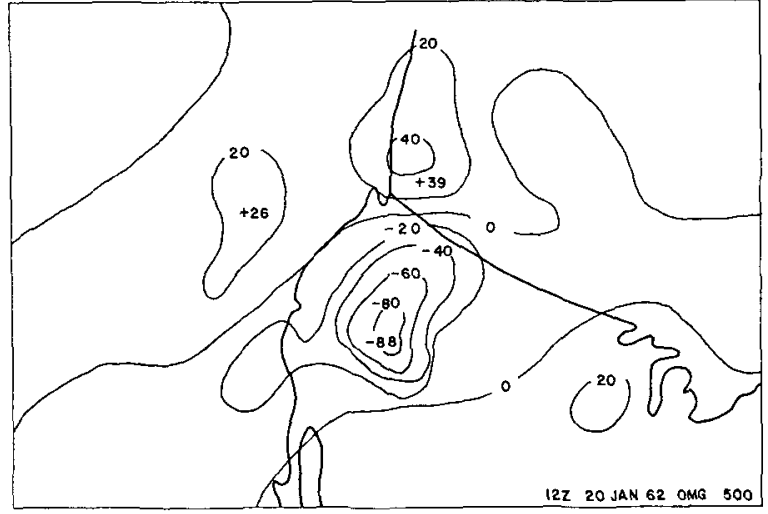

FIG. 17. 500-mb $\omega$ field $\left(10^{-4} \mathrm{mb} \mathrm{sec}^{-1}\right)$ for $1200 \mathrm{GCT}$, 20 January 1962

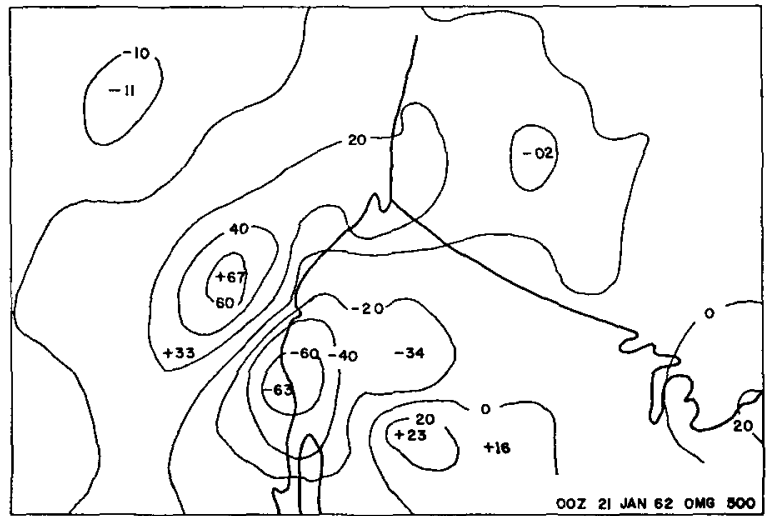

Fig. 19. $500-\mathrm{mb} \omega$ field $\left(10^{-4} \mathrm{mb} \mathrm{sec}^{-1}\right)$ for $0000 \mathrm{GCT}$, 21 January 1962.

sionally up to about a dozen units, which could amount to 50 per cent of the $f_{M}{ }^{2}$ value.

\section{E. Relationship between computed vertical velocities and prevailing weather}

The large-scale vertical velocity field, as such, is perhaps of little interest to the typical weather forecaster; rather its value lies mainly in its relationship to precipitation, cloudiness, visibility, etc. As part of this investigation the vertical velocity fields were computed during the period of the severe west coast storm occuring shortly after mid-January. A wave developed on a front. oriented from northwest to southeast in northwestern United States and subsequently moved south to southeast. Rain began in the Monterey area about $1500 \mathrm{GCT}$ 19 January and continued through 1300 GCT 20 January. The front passed Monterey about 1000 GCT 20 January; however no appreciable pressure rise occurred for about $48 \mathrm{hr}$. Intermittent showers continued to about 1800 GCT 20 January.

Unfortunately, the machine-analyzed data was not available for 19 January and 0000 GCT 20 January. Figs. 16 and 17 for 1200 GCT 20 January show that by 


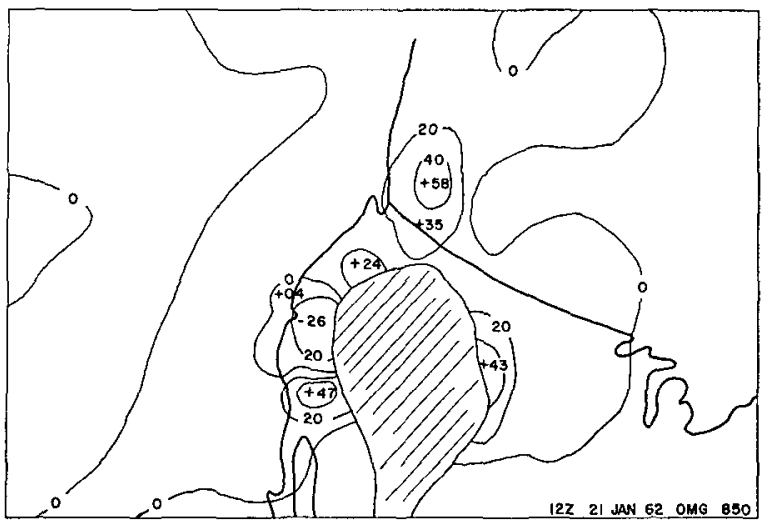

FIG. 20. 850-mb $\omega$ field $\left(10^{-4} \mathrm{mb} \mathrm{sec}^{-1}\right)$ for $1200 \mathrm{GCT}$, 21 January 1962.

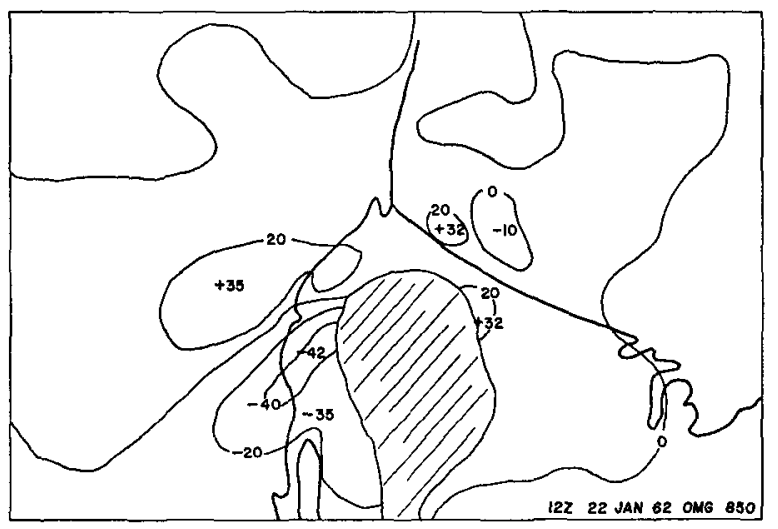

FIG. 22. 850-mb $\omega$ field $\left(10^{-4} \mathrm{mb} \mathrm{sec}^{-1}\right)$ for $1200 \mathrm{GCT}$, 22 January 1962.

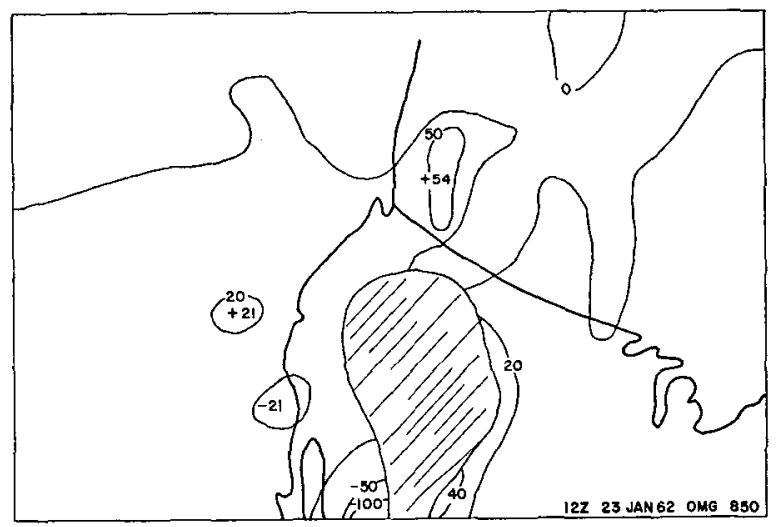

FIG. 24. 850-mb $\omega$ field $\left(10^{-4} \mathrm{mb} \mathrm{sec}^{-1}\right)$ for $1200 \mathrm{GCT}$, 23 January 1962 .

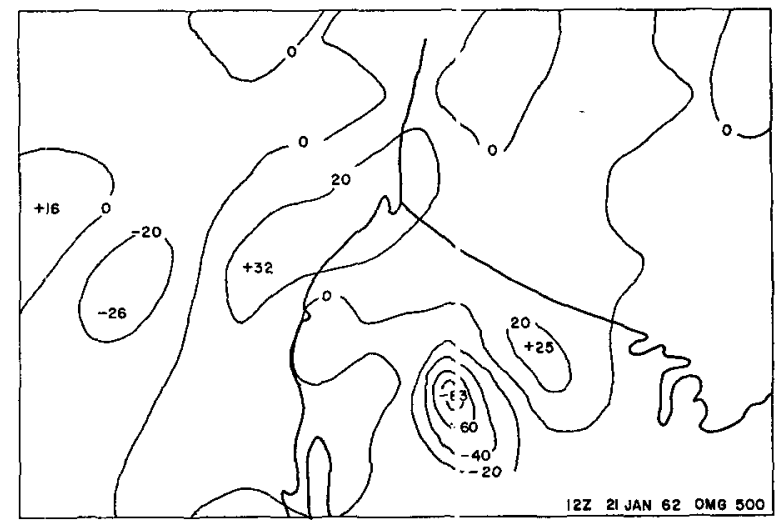

FIg. 21. $500-\mathrm{mb} \omega$ field $\left.\left(10^{-4} \mathrm{ml}\right) \mathrm{sec}^{-1}\right)$ for $1200 \mathrm{GCT}$, 21 January $1,62$.

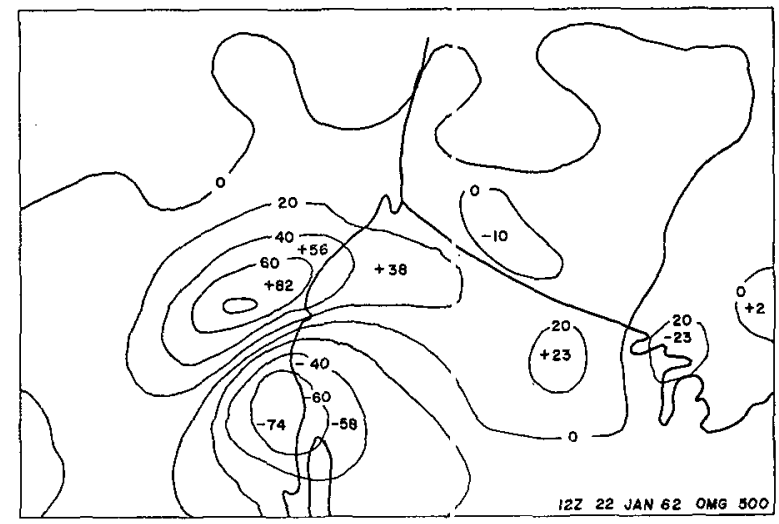

FIG. 23. 500-mb $\omega$ field $\left(10^{-4} \mathrm{mb} \mathrm{sec}^{-1}\right)$ for $1200 \mathrm{GCT}$, 22 January 1962.

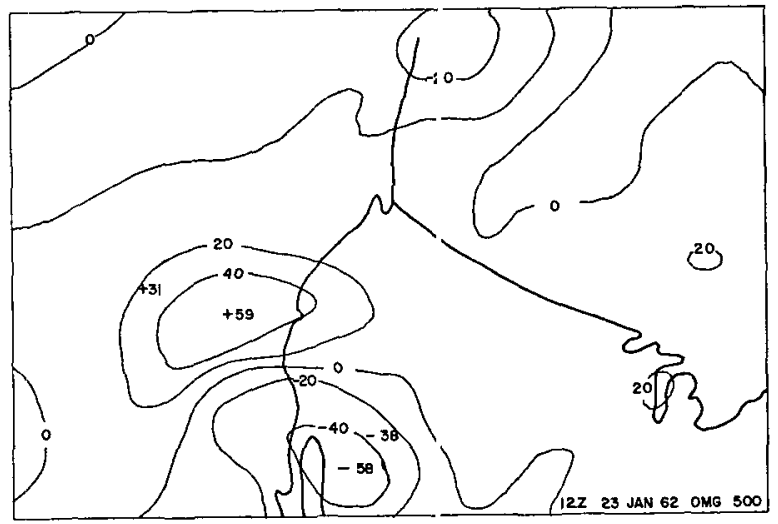

FIG. 25. 500-mb $\omega$ field $\left(10^{-4} \mathrm{~m} . \mathrm{b} \mathrm{sec}-1\right)$ for $1200 \mathrm{GCT}$, 23 January : 962. 


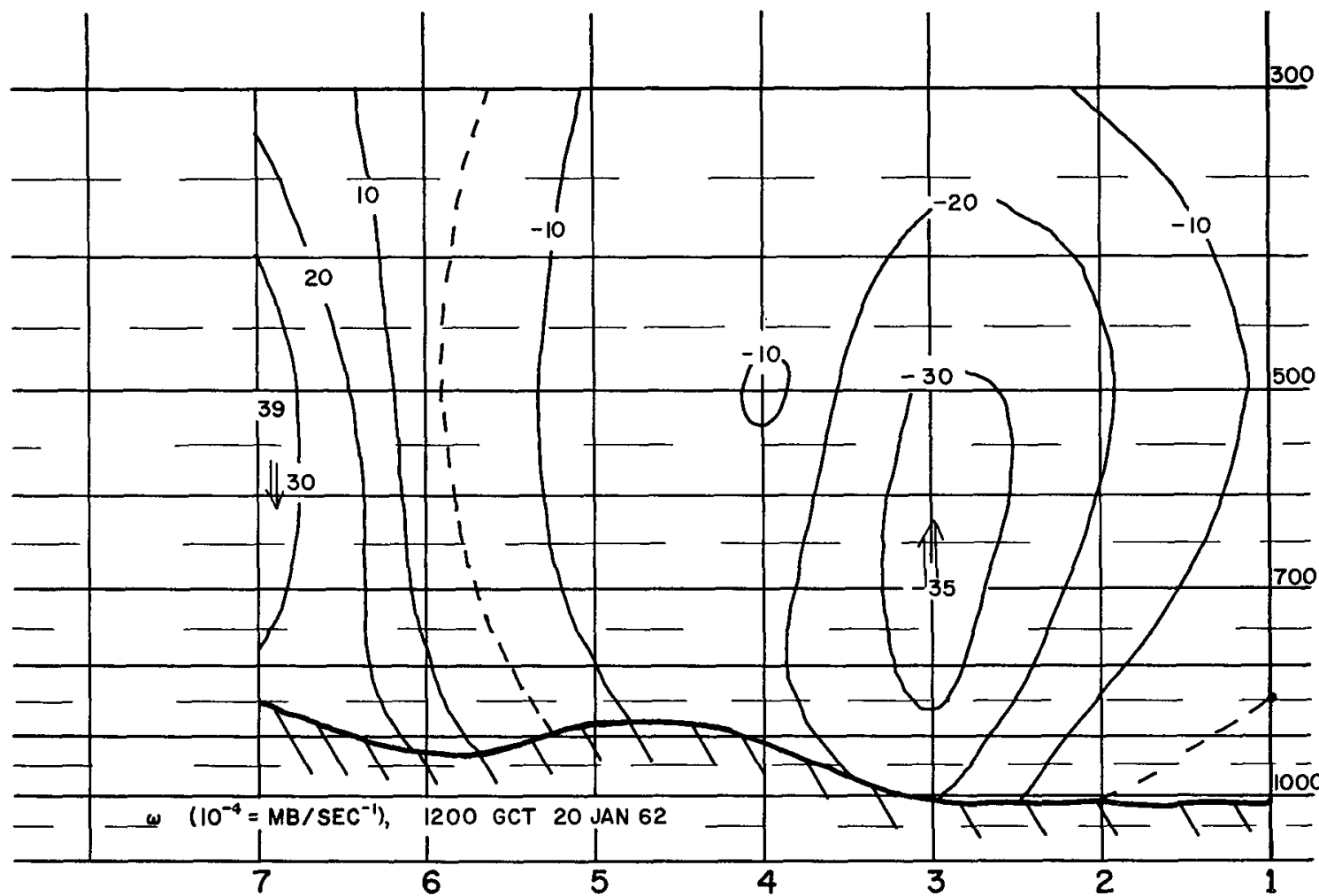

Fia. 26. Cross section (along heavy line in Fig. 9) showing vertical velocity $\omega\left(10^{-4} \mathrm{mb} \mathrm{sec}^{-1}\right)$ versus $\ln p$ for $1200 \mathrm{GCT}$, 20 January 1962.

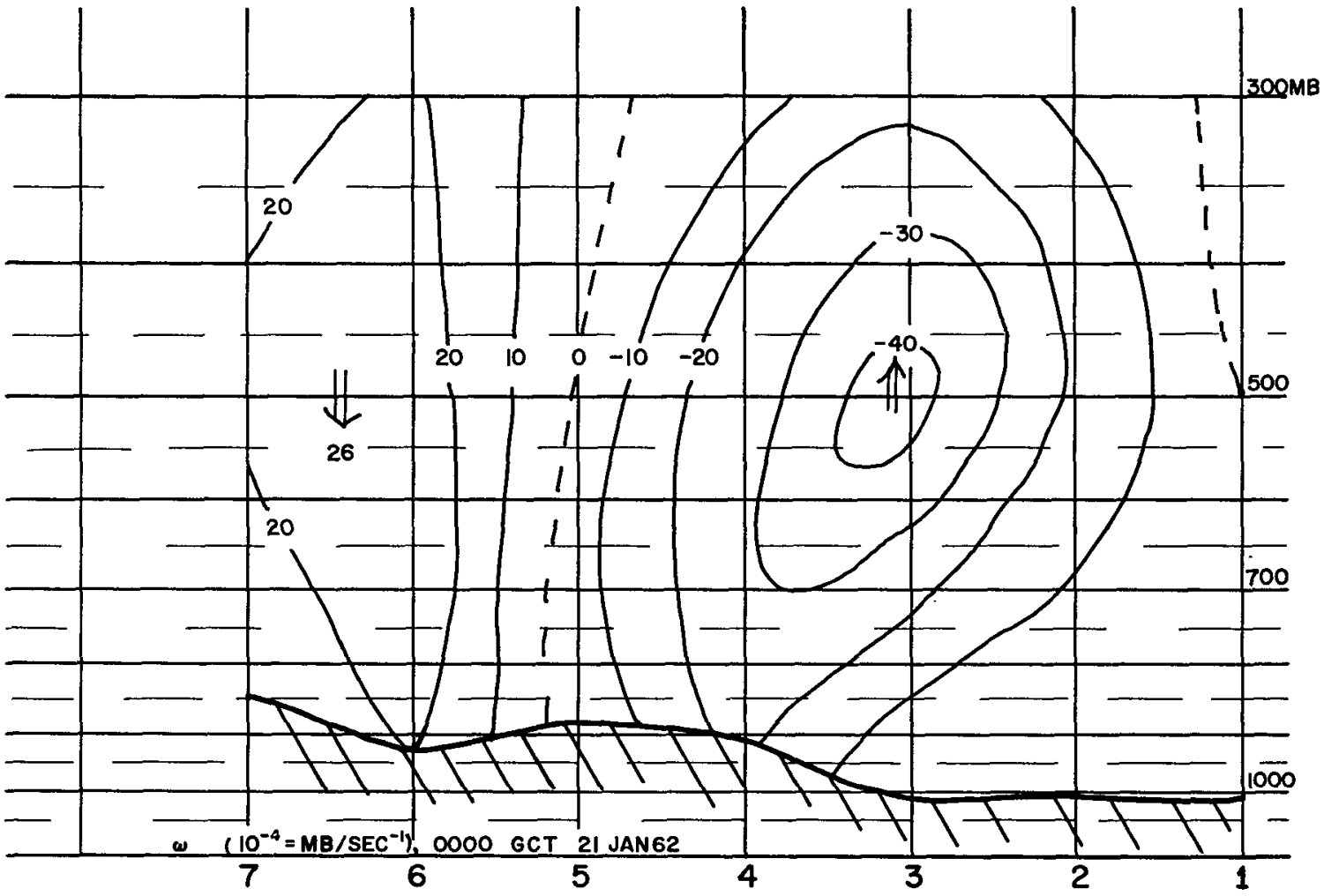

FIG. 27. Similar to Fig. 26 for 0000 GCT, 21 January 1962. 


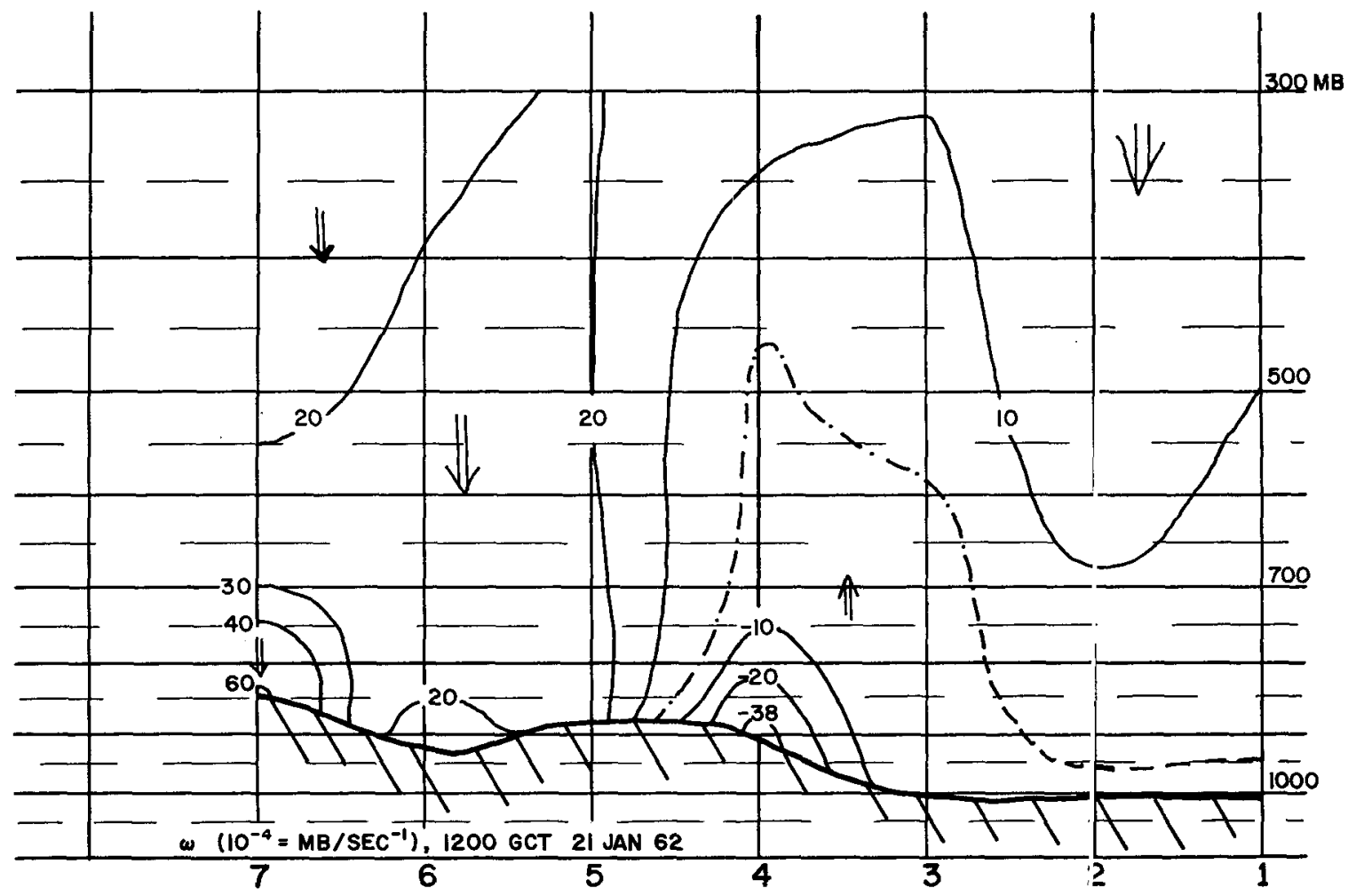

FIG. 28. Similar to Fig. 26 for 1200 GCT, 21 January 1962.

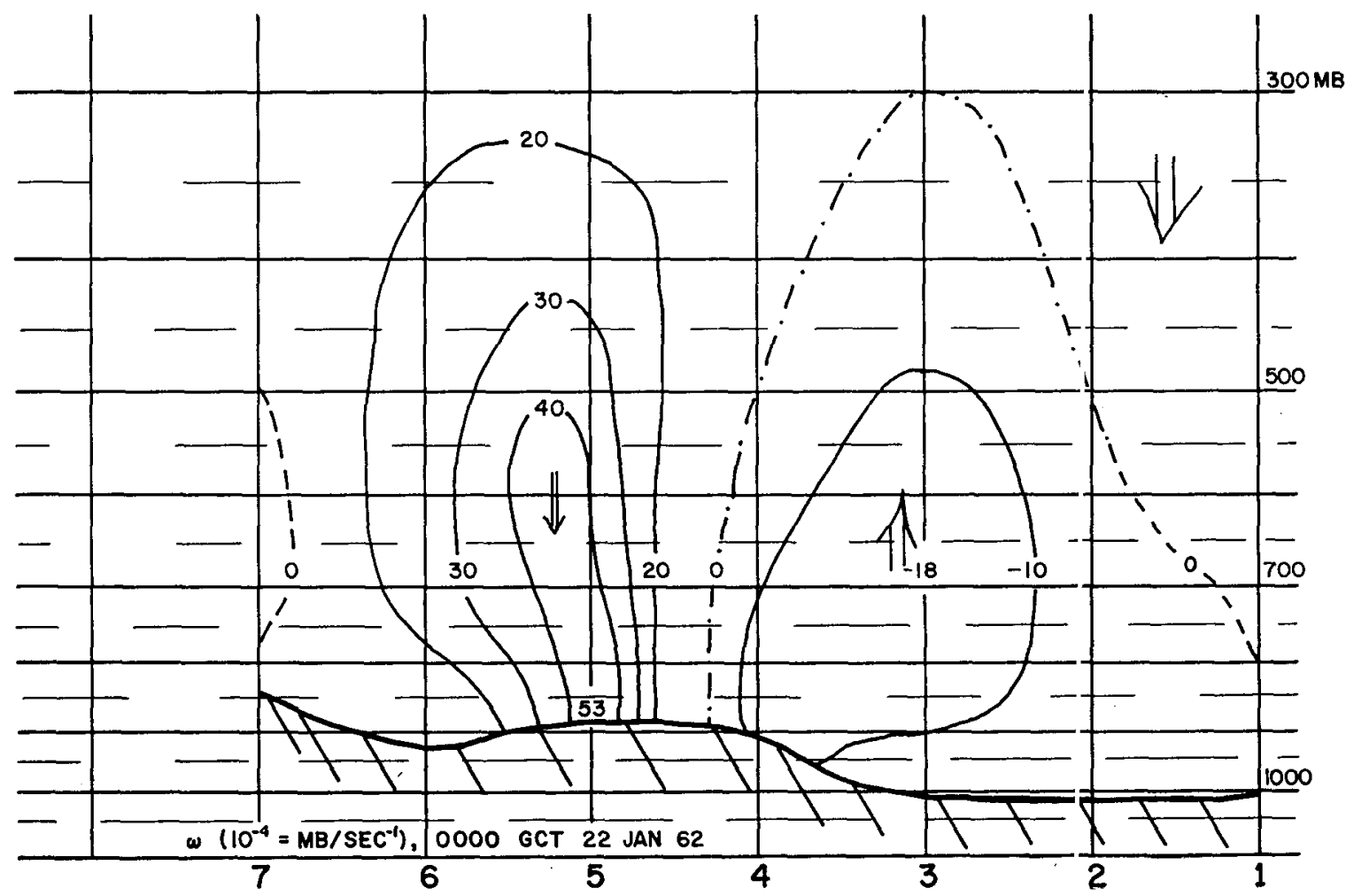

FIG. 29. Similar to Fig. 26 for 0000 GCT, 22 January 1962. 


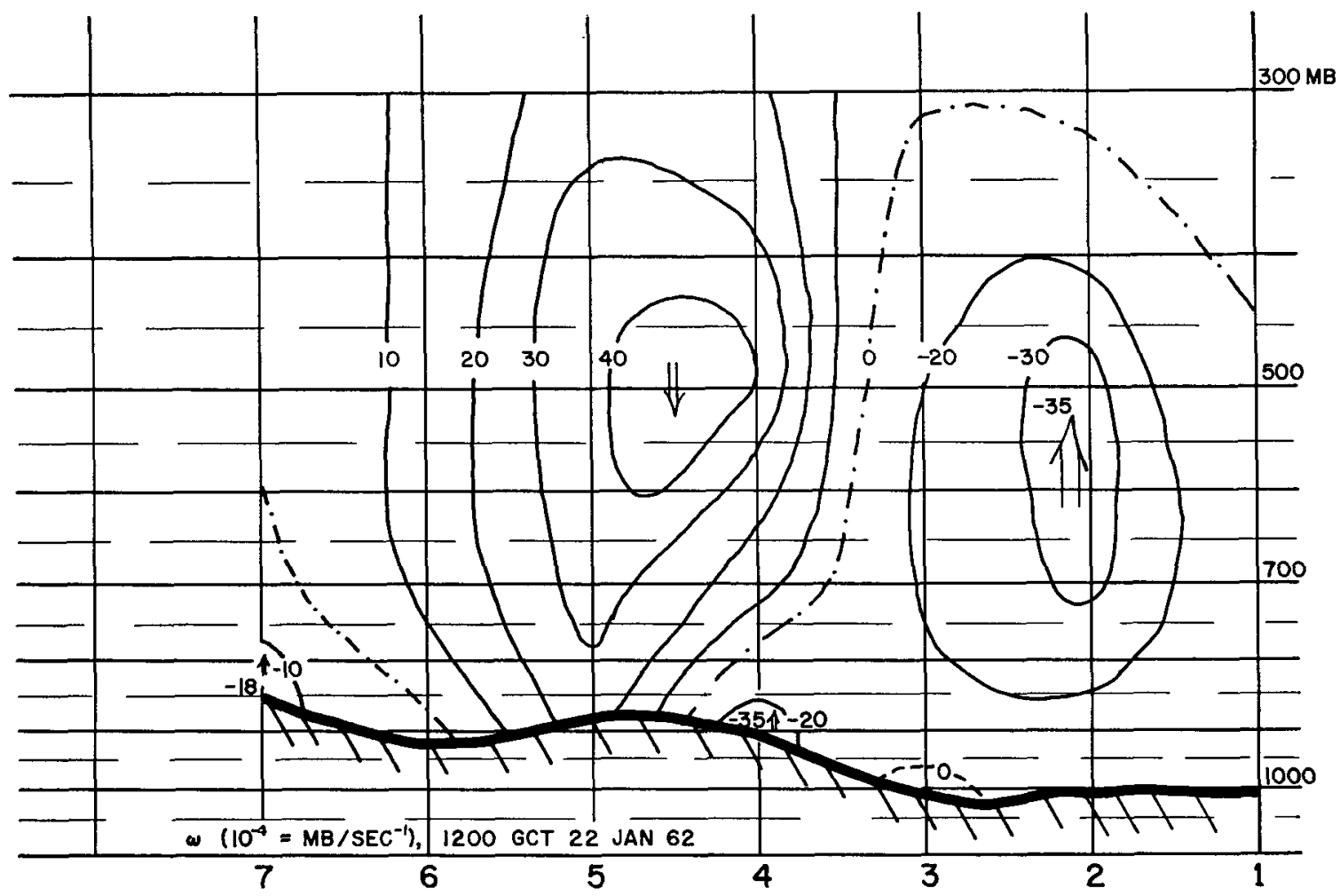

FIG. 30. Similar to Fig. 26 for 1200 GCT, 22 January 1962.

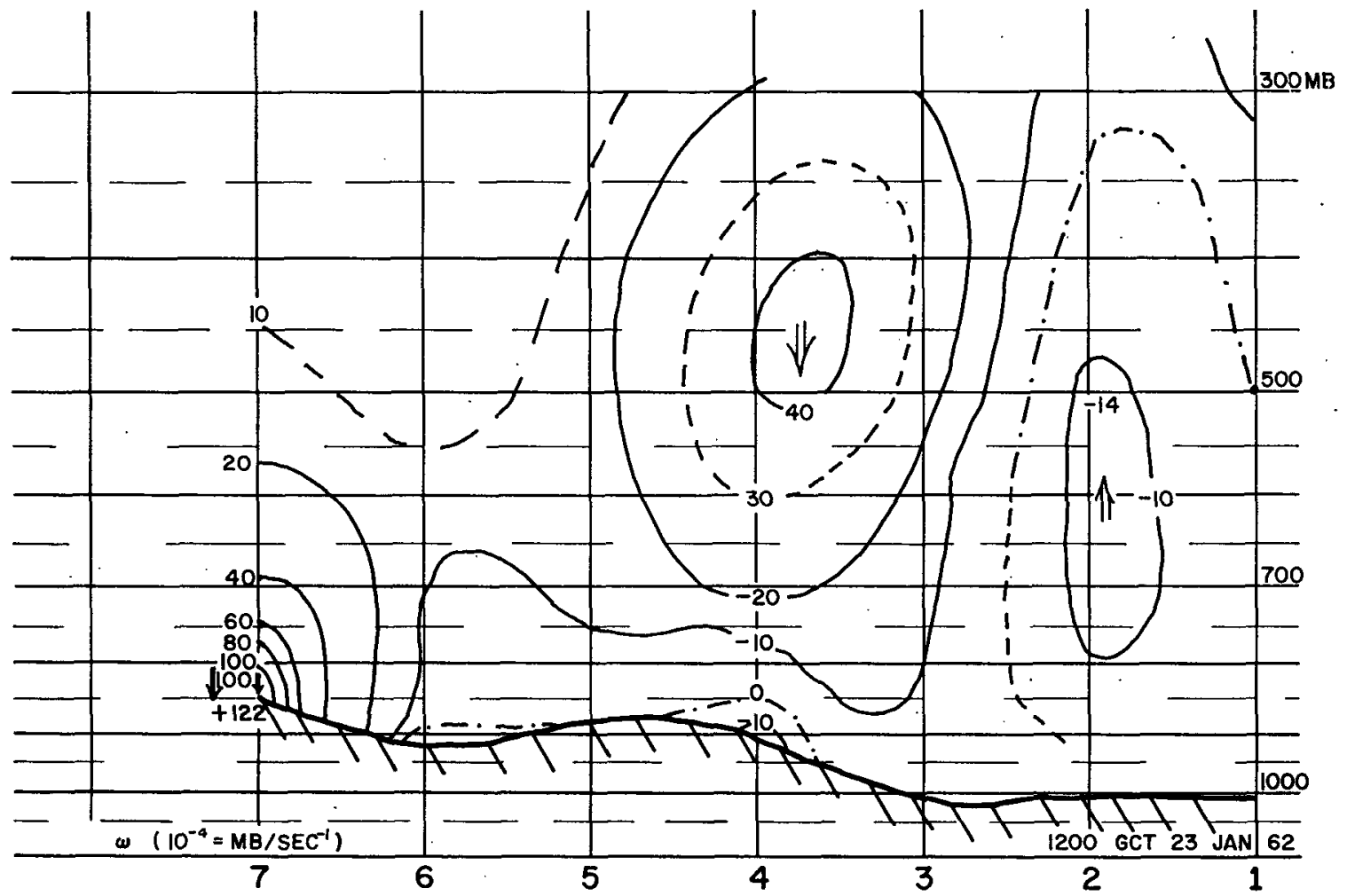

Fig. 31. Similar to Fig. 26 for 1200 GCT, 23 January 1962. 


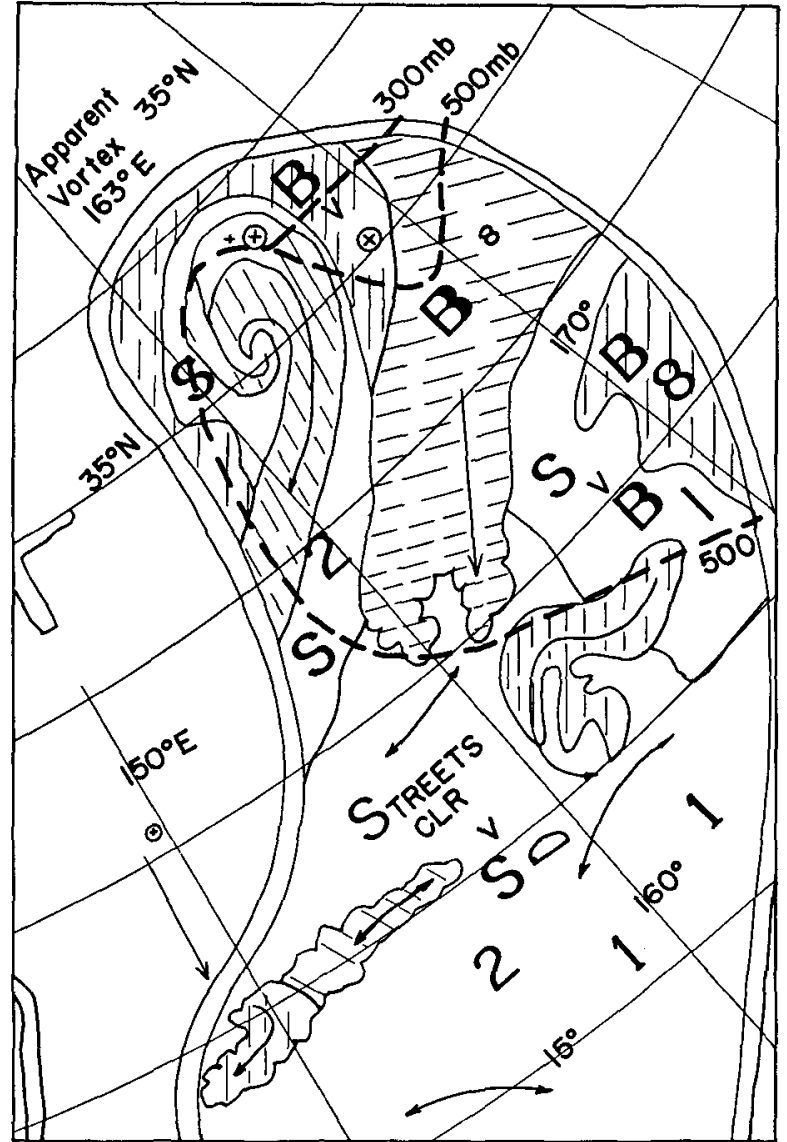

FIG. 32. A TIROS cloud picture for a Pacific Ocean Area for 0500 GCT, 17 May 1962. Dashed lines indicate computed upward motion at 500 and $300 \mathrm{mb}$.

this time the maximum vertical velocities lie generally east of Monterey, which lies between points 3 and 4 on the heavy line shown on Fig. 16. Subsiding air is found off the coast to the west. By 0000 GCT 21 January (Figs. 18 and 19), the subsidence had increased at 500 $\mathrm{mb}$ but had not moved eastward. In fact upward velocities persisted over Monterey, although the main area of ascending motion moved somewhat southward. Rain commenced again locally about 0600 GCT 21 January, with snow pellets by 1400 and snow showers by 1615 . Snow and rain were frequent until 1000 GCT 22 January, mainly from cumulus with tops generally under $15,000 \mathrm{ft}$. Calculation of the static stability parameter $\sigma$ showed a minimum of stability near the Monterey area. Ascending motion, primarily at low levels (see Figs. 18, 20 and 22), continued during the period of precipitation; however slightly descending motion may be noted above $500-\mathrm{mb}$ level from 1200 GCT 21 January through 1200, 22 January (see Figs. 21, 23 and 25), increasing with time up through 1200, 23 January. By the latter time, descending currents prevail throughout the local area.
It appears, therefore, that the precipitation during the 21 st and 22 nd was primarily a result of low-level vertical motions, strongly affected by topography, during a period of relative instability. These terrain effects were not reflected in the National Meteorological Center vertical velocities for $600 \mathrm{mb}$. However, the latter, with some important exceptions, wers in fairly good agreement with the vertical velocities near mid-troposphere as computed with this model.

Some further insight regarding the vertical velocity field may be gained from north-sciuth cross sections constructed along the line shown in Fig. 16. Note, for example, from Figs. 26 to 31 tha: the maximum magnitude of the vertical velocity may occur at almost any level from the earth's surface to above the 500 -mb level. Observe also the presence of ascending motion of varying intensity, sometimes only at low levels, in the Monterey area on all cross sections from $1200 \mathrm{GCT}$ 20 January up to the last chart of 1200 GCT 23 January where subsidence prevails.

The reliability of the computed vertical velocity fields over areas of sparse data is a matter of considerable interest. With cloud data now available from TIROS satellites, there exists the opportunity to make some sort of comparison of these two parameters. Fig. 32 shows a randomly chosen TIROS picture over the Pacific Ocean for 0500 GCT 17 May 1962. The superposed dashed line delineates the area of computed upward motion at the 500- and $: 00$-mb levels for 1200 GCT 17 May and shows good agreement with the cloud area.

\section{Summary and conclusions}

An $\omega$-equation has been solved over a three-dimensional Northern Hemisphere grid at 850, 700, 500, and $300 \mathrm{mb}$ with a lower-boundary condition which is applied at terrain pressure and includes effects of frictionally- and terrain-induced vertical velocities. The latter, as computed here, had smaller maximum magnitudes and less irregularity, or smaller scale features, than the frictionally-induced vertical velocity.

The calculations showed that the terrain and friction effects markedly influence the $\omega$-fields in the lower troposphere but largely disappiaar by $500 \mathrm{mb}$ for the rather heavily smoothed mount:ins used in the current experiments. .

Computations with several ;:tatic stability parameters, namely, a constant valus: $\overline{\bar{\sigma}}$, a pressure-variable value $\bar{\sigma}$ and a point-variable value $\sigma$, exhibited the greatest differences in the maximum $\omega$ 's, as much as 50 per cent, at $300 \mathrm{mb}$ particuliırly between the $\overline{\bar{\sigma}}$ case and the other two. At lower levels the differences are only about 10 per cent. This appears comparatively small as far as the synoptic aspects are concerned; however the cumulative effect might be significant if the $\omega$-fields were incorporated into an iterative scheme for the time integration of the equetions of motion. 
Similarly when computations were made utilizing the term $f \eta$ versus $f_{M^{2}}$ in the coefficient of the derivative $\partial^{2} \omega / \partial p^{2}$, differences up to 50 per cent of the $f_{M}{ }^{2}$ values occasionally occurred in the centers of maximum vertical velocity; but mostly the differences were only 10 to 25 per cent.

The computed vertical velocity fields for the period of a severe winter storm on the west coast of the United States appear to show a good correlation with the weather and indicate the desirability of including the lower-boundary influences of friction and irregular terrain.

\section{REFERENCES}

'Arnason, G., 1956: Convergence rates of Liebmann's and Richardson's iterative methods when applied to the solution of a system of Helmholtz-type equations. Tech. Memo No. 10, Joint Numerical Weather Prediction Unit, U. S. Weather Bureau, 26 pp.
Bellamy, J. C., 1945: The use of pressure altitude and altimeter corrections in meteorology. J. Meteor., 2, 1-80.

Berkofsky, L., and E. A. Bertoni, 1960: Topographic charts at one-degree intersections for the entire earth. GRD Research Notes, No. 42, Geophysics Research Directorate, U. S. Air Force, Bedford, Mass., 43 pp.

Burnett, F. W., 1961: Synoptic meleorology as practiced by the National Meteorological Center. U. S. Government Printing Office, Washington, D. C., 73 pp.

Cressman, G. P., 1960: Improved terrain effects in barotropic forecasts. Mon. Wea. Rev., 88, 327-342.

Haltiner, G. J., and F. L. Martin, 1957: Physical and dynamical meteorology. New York, McGraw-Hill, $470 \mathrm{pp}$.

Lorenz, E. N., 1960: Energy and numerical weather prediction. Tellus, 12, 364-373.

Salvadori, Mario G., and Melvin L. Baron, 1952: Numerical methods in engineering. New York, Prentice Hall, 258 pp.

Sawyer, J. S., 1959: The introduction of the effects of topography into methods of numerical forecasting. Quart. J.R. meteor. Soc., 85, 31-43.

Wiin-Nielsen, A., 1959: On certain integral constraints for the time-integration of baroclinic models. Tellus, 11, 45-59. 